\title{
Systematic Study of Important Variables in Absorption Drug Loading into Specially Formulated Ion-Pairing PLGA Nanoparticles Using Doxorubicin Hydrochloride as Model Drug
}

\author{
Korlapativenkateswara Rao
}

\begin{abstract}
An effective drug loading procedure should be simple and time-efficient to maximize drug loading or uptake: adsorption drug loading, when optimized, could provide an important tool to fulfill these requirements. With this in mind, the purpose of this study was fourfold. First, evaluate the ion pairing potential between borate-based lipophilic anions and a model cationic drug, doxorubicin hydrochloride (doxHCl), in organic solvents, to introduce attractive force for absorption loading. Second, formulate blank polymeric nanoparticles incorporating borate-based lipophilic anions using oil-in-water $(O / W)$ emulsion for adsorption drug loading. Third, systematically study and optimize absorption loading of doxHCl into the aforementioned nanoparticles by analyzing different variables. Lastly, having optimized the drug uptake conditions, evaluate doxHCl uptake by nanoparticles in plasma.

Results shown an almost 1:1 molar attraction between lipophilic anions and doxHCl as well as successful formulation of PLGA or PLGA/PEG-PLA polymeric nanoparticles incorporating one of the borates, potassium tetrakis(4-chlorophenyl) borate (KTpCIPB). These nanoparticles were spherical and XPS confirmed the presence of PEG polymer on their surface. Adsorption drug loading was optimized using polymers with high acid number $(502 \mathrm{H})$, maximizing KTpCIPB incorporation, increasing incubation/uptake temperature, increasing drug: nanoparticle incubation ratio, and using PEGylated nanoparticles (those containing PEGPLA). Under optimized conditions, doxHCl loading reached as high as 6\%. Studies with another model drug, vinblastine sulfate(VS), confirmed the utility of this loading procedure and specially formulated nanoparticles. Plasma drug uptake study showed marginal reduction of plasma drug concentrations near the normal therapeutic dose of doxHCl while the nanoparticle system effectively reduced drug concentration by almost $16 \%$, when plasdma drug concentration was 4 times the normal therapeutic dose $(200 \mu \mathrm{g} / \mathrm{ml})$. favourable results supported the use of borate-encapsulated polymeric nanoparticles for improved model drug loading via absorption/extraction loading procedure under different conditions.
\end{abstract}

\section{Introduction}

Nanoparticulate polymeric drug delivery systems have been the subject of intense research due to advantages including extended blood circulation time, imporved passive or active drug ttargeting, reduce side effects and wasted drug, prolonged drug release for extended dosing intervals, and biocompatibility of different biodegradable polymers available for nanoparticles formulation (Soppimath, Aminabhavi et al.2001; Yang, Wanget al.2006; Park 2007).

An important feature of these systems is drug loading, which may help to directly reduce total drug amount per dose and total dosing time while indirectily improving total release priod as well as drug accumulation at target sites. For polymeric nanoparticles, many drug loading procedures have been established to improve loading of different drugs, each with their unique properties. Two commonly cited strategies are drug entrapment, where drug molecules are trapped in the hydrophobic polymer matrix of nanoparticles during formulation, and drug conjugation, where drug molecules are covalently linked to polymer strands before or after nanoparticle formulation. Three commonly cited entrapment methods are emulsification/solventevaporation, nanoprecipitation, amd polymerization. Emulsification/solvent-evaporation method takes advantage of phase separation between oil and water to promote homogenization/sonication-driven nanoparticle formation and examples include oil-in-water $(\mathrm{O} / \mathrm{W})$ or water-in-oil-in-water (W/O/W) emulsions. Nanoprecipitation method involves partitioning of water-soluble oil phase into an aqueous phase to promote emulsification without power input. The polymerization method, as the name suggests, is where nanoparticles are prepared through chemical polymerization of monomers (Soppimath, Aminabhavi et al. 2001; GalindoRodriguez, Allemann et al. 2004). These different strategies differ in that each may be ;tailored to maximize loading of ; different types of drugs as well as facilitate the ;use of special polymersx (Zoble, Zimmer et al. 1999: Krauel, Davies et al. 2005; Arias, Ruiz et al.2008). despite the effectiveness of entrapment methods, drug release from these nanoparticles have shown a large initial burst with the first 1-3 $\mathrm{h}$ of release and minimal release afterwards (Some, Dubernet et al. 2000; Wong, Bendayan et al.2004; Missirlis, Kawamura et al. 2006). To overcome this, drug conjugation can be utilized where drug molecules are chemically linked to polymer 
strands followed by use of these conjugates for particle formulation or linked to pre-formed nanoparticles (Yoo,Oh et al. 1999). Even though zero-order drug release can be achieved through hydrolysis-limiting drug release, drug loading is low due to limited availability of conjugation sites.

Another strategy now more commonly used to complement entrapment and conjugation loading methods is the absorption method, which as the name suggests, achieves drug loading though incubation of blank nanoparticles with a concentrated drug solution (Asuri, Karajanagi et al. 2007; Arias, Linares-Molinero et al. 2008). This method originates from the need to encapsulate protein/peptide drugs into naoparticles, where drugs could lose efficacy during stressful entrapment and conjugation loading procedures while the more passive adsorption loading process preserved drug intergrity and efficacy. In studies cited thus far, the passive drug concentration gradient and potential hydrophobic interactions between the polymer and protein/peptide have played important roles in imporving drug loading.

Recently, the concept ofion pairing has been incorporated into nanoparticles undergoing adsorption loading. Here, electrostatic interactions are intergrated into nanoparticles, by using charged polymers to formulate charged nanoparticles,to provide additional attractive forces with oppositely charged drug molecules.examples of the above strategy include using sulfobutylated poly(vinyl alcohol)-graft-poly(lactideco-glydolide) to incorporated tetanus toxoid(Jungm Breitenbach et al. 2000), poly( $\epsilon$-caprolactone) poly(ethylene glycol)-poly( $\epsilon$-caprolactone) to enhance human basic fibroblast growth factor incorporation (Gou, Huang et al. 2007) and using poly (styrene-co-4-styrene-sulfonate) to enhance lysozyme loading (Cai, Bakowsky et al. 2008). Existing absorption drug loading procedures incorporating ion complexation or ion pairing, despite their effectiveness, still has several shortcomings. One is that charged polymers may be difficult to synthesize, purify, and characterize. Second, the potential utility of absorption loading for small molecule drugs, especially cationic anti-cancer drugs such as doxorubicin hydrochloride and vinblastine sulfate, have not been systemically evaluated, especially compared to existing ones. Third, no existing studies have evaluated the effectiveness of PEGylated nanoparticles, those with a layer of hydrophilic poly(ethylene glycol) (PEG) polymer on the surface, for absorption drug loading. Lastly, alternative uses of charged polymeric nanoparticles have not been explored.

With the above issues in mind, there are several goals for this study. First, evaluate the ability of borate-based lipophilic anions to extract a model cation drug, doxorubicin hydrochloride, from aqueous to the organic phase. Second, a new anionic polymeric nanoparticle will be formulated using an FDA-approved polymer, PLGA, and borate-based lipophilic anions and properties such as borate incorporation, particle size and morphology will be evaluated. Third, absorption loading of doxHCl by the specially formulated nanoparticles will be studied systemically to optimize loading. Fourth, PEGylated nanoparticles will be formulated to evaluate its absorption drug uptake potential. Lastly, drug uptake in plasma will be explored to evaluate alternative uses for this nanoparticle system.

\subsubsection{Materials}

\section{Material and Methods}

Potassium tetrakis(2-thienyl) borate (KTTB), potassium tetrakis(4-chorophenyl) borate (KTpCIPB), and potassium tetrakis[3,5-bis (trifluoromethyl)phenyl]borate (KTBTFMPB) were purchased from Sigma-Aldrich Sodium tetraphenylborate (NaTPB) was ind gift from Dr.Mark Meyerhoff. Doxorubicin hydrochloride (doxHCL) (>99\% purity, lyophilized powder) was purchased from Hisun Pharmaceuticals (China). Poly (D,Llactide-co-glycolide), 50/50, with inherent viscosity of $0.2 \mathrm{dl} / \mathrm{g}$ and $\mathrm{MWw}$ of $15 \mathrm{kDa}$ (resomer $502 \mathrm{H}$ ), $0.34 \mathrm{dL} / \mathrm{g}$ and MWw of $38 \mathrm{kDa}$ (resomer $503 \mathrm{H}$ ), and $0.42 \mathrm{dL} / \mathrm{g}$ and $\mathrm{MWw}$ of $45 \mathrm{kDa}$ (resomer 503), were purchased from Boehringer Ingelheim. Poly(vinyl alcohol) (PVA) with MWw of 25,000 (88\% hydrolyzed) was purchased from Polysciences Inc. L-lactide ( $>99 \%$ purity) was purchased from Sigma-Aldrich. Methoxy-poly(ethylene glycol) (mPEG-NH2, MWw=5,000) was purchased from Nektar Therapeutics. Spectra/por 7 dialysis bags (MWCO; 10$12 \mathrm{~K}$ or $50 \mathrm{~K}$, Spectrum) were purchased from Fisher Scientific All other chemicals and solvents were of analytical grade and purchased from commercial suppliers.

\subsubsection{Borate solubility}

Borates were weighed into glass tubes followed by addition of different organic solvents in $0.5 \mathrm{~mL}$ incremental volumes at room temperature. After shaking for $30 \mathrm{~s}$, solubility was determined as the concentration when the solution was completely clear.

\subsubsection{DoxHCl extraction into borate-dissolved ethyl acetate phase}

DoxHCl was dissolved in deionized water (DIW) at $1.72 \mathrm{mM}$. KTTB, KTpCIPB, and KTBTFMPB were dissolved in ethyl acetate at $0.5: 1,1: 1$, or 2:1 molar ratios with doxHCl. For extraction, $1 \mathrm{~mL}$ of either blank or borate-dissolved ethyl acetate solution was added to $1 \mathrm{~mL}$ doxHCl solution followed by vortexing for $30 \mathrm{~s}$ and shaking for 10min. the mixture was centrifuged at 5,000 RPM for $5 \mathrm{~min}$, and the doxHCl concentration in the aqueous phase before and after extraction was determined by HPLC (see below. 
Percent doxHCl extraction was calculated as:

$\%$ Drug Extraction $=\quad$ DoxHCl Conc before extraction - DoxHCl Conc after extraction $\times 100$

\author{
DoxHCl Conc before extraction
}

\title{
3.3.4 DoxHCl concentration determination methods \\ 3.3.4.1 HPLC method
}

A Waters HPLC system (Waters, Milford, MA) was used for chromatographic separation of doxHCl. It consisted of a 1525 Binary HPLC pump, 717 plus autosampler, and 474 scanning fluorescence detector. Waters Breeze chromate-graphy software was used to acquire and process data. A Waters Nova-Pak C18 column (3.9 x $150 \mathrm{~mm}$ I.D.) (Waters, Milford, MA) was used with a filtered and degassed ACN : 20mM, pH 3 potassium phosphate : TFA (30:70:0.1, v/v/v) mobile phase. Chromatography was performed at a flow rate of $1 \mathrm{~mL} / \mathrm{min}$ and fluorescence detection was set at גexcitation of $480 \mathrm{~nm}$ and גemission of $590 \mathrm{~nm}$ at room temperature.

$\mathrm{DoxHCl}$ in the samples were analyzed using corresponding standards injected separately.

\subsubsection{UV method}

A Beckman DU650 spectrophotometer was used for UV analysis. Samples or standards containing doxHCl in analyzed using a quartz spectrophotometer cell (Starna Cells Inc.) at a scanning rate of 1200/min and scanning range of $400-750 \mathrm{~nm}$. Absorbance wavelength was set at $480 \mathrm{~nm}$ and all sample had baseline reading set at $750 \mathrm{~nm}$.

\subsubsection{PEG-PLA copolymer synthesis and characterization}

Methoxy-poly(ethylene glycol)-poly(lactic acid) (PEG-PLA) was synthesized by a standard ring opening polymerization. Briefly, L-lactide (Sigma, 98\% purity) was pre-purified by recrystallization from ethyl acetate and the catalyst, stannous octoate (Tin(II) 2-ethylhexanoate, Sigma, 95\% purity), was pre-purified by distillation. The desired molar ratio of L-lactide to $\mathrm{mPEG}-\mathrm{NH} 2$ was weighed out and added to the reaction flask followed by addition of stannous octoate at equivalent molar ratio to $\mathrm{mPEG}-\mathrm{NH} 2$. Dry toluene $(10 \mathrm{~mL} / 1.5 \mathrm{~g}$ of reactants) was added to dissolve and mix the reagents. The reaction proceeded with stirring at 110C for 2 hours under moisture-free argon atmosphere. At the end of the polymerization step, copolymer was purified and recovered as follows. The copolymer crystallized upon cooling and was dissolved with cooled dicholoromethane followed by ether addition to reprecipitate the copolymer. The previous procedure was repeated and the copolymer was collected by filtration on a Buchner funnel. They were then dried at 37C in a vacuum oven overnight.

PEG-PLA was analyzed by 1H-NMR spectroscopy using a Bruker DRX 500 spectrometer (Bruker Biospin Inc., Billerica, MA) operating at $500 \mathrm{MHz}$. Peak areas for different hydrogens were used for PEG-PLA molecular weight determination.

\subsubsection{General nanoparticle formulation}

Nanoparticles were formulated using an oil-in-water $(\mathrm{O} / \mathrm{W})$ emulsion/solvent evaporation procedure (Fig.3.6). Different amounts of polymer were dissolved in $2 \mathrm{~mL}$ ethyl acetate (oil phase) and emulsified by sonication $(20 \mathrm{~s}$ ) on ice using a probe sonicator (Sonics VibraCell) at $50 \%$ power in $2 \mathrm{~mL} 3 \%$ PVA solution (aqueous or water phase). The $\mathrm{O} / \mathrm{W}$ emulsion was diluted into $40 \mathrm{~mL}$ of $0.075 \%$ PVA solution under rapid magnetic stirring for 4-5 hours. Nanoparticle suspensions were collected and washed using Amicon Stirred Cell with a $50 \mathrm{~nm}$ Millipore nitrocellulose filter.

KTpCIPB-loaded nanoparticles were formulated using the same O/W procedure above except that predetermined amounts of KTpCIPB were dissolved in the ethyl acetate phase along with polymer.

PEGylated nanoparticles were formulation using the same $\mathrm{O} / \mathrm{W}$ procedure above with modifications. $502 \mathrm{H}$ PLGA and KTpCIPB were dissolved in $2 \mathrm{~mL}$ acetate and mixed with PEG-PLA dissolved in $0.2 \mathrm{~mL}$ methylene chloride. This oil phase was emulsified in $2.2 \mathrm{~mL}$ of $3 \%$ PVA solution. All other steps were unchanged.

\subsubsection{Nanoparticle characterization}

\subsubsection{Particle size/zeta potential determination}

Nanoparticle suspensions were diluted to $0.5 \mathrm{mg} / \mathrm{mL}$ and transferred into fold capillary cells for both zeta potential and particle size determination. Samples were analyzed using a Malvern ZetaSizer Nano $\mathrm{ZSv}($ Malvern Instruments.

\subsubsection{SEM morphological analysis}

Nanoparticle suspensions were diluted to $50 \mu \mathrm{g} / \mathrm{mL}$ and dried overnight on viewing stubs. Morphology was observed by scanning electron microscopy (Philips XL 30 Field Emission Gun Scanning Electron Microscopy). 


\subsubsection{Surface chemistry analysis}

Surface chemistry of nanoparticle with or without PEG-PLA (no drug loaded) as well as PEGylated nanoparticles with doxHCl loaded was characterized by X-ray photoelectron spectroscopy (XPS, Kratos Axis Ultra, Kratos Analytical) equipped with a monocromatized aluminum X-ray source (powered at $10 \mathrm{~mA}$ and 14 $\mathrm{KV})$. The angle between the normal to the sample surface and the lens axis was $\mathrm{O}^{\circ}$. A survey scan spectrum $(0-$ $1000 \mathrm{eV}$, average of 4 scans) and regional scan for C1s (265-295 eV, average of 10 scans) were recorded for each sample. The analyzer was set at pass energies of $160 \mathrm{eV}$ or $20 \mathrm{eV}$ for the survey or regional scans, respectively. Analysis of data was done with CasaXPS program (Casa Software Ltd., UK) with a Gaussian/Lorentzian product function. Chemical shifts for the $\mathrm{C} 1 \mathrm{~s}$ regional scans were referenced to hydrocarbon at $285 \mathrm{eV}$.

\subsubsection{KTpCIPB loading determination}

KTpCIPB was extracted from nanoparticles by adding $0.1 \mathrm{~mL}$ methylene chloride followed by $1.5 \mathrm{~mL}$ methanol to pre-weighed nanoparticle powder. The suspension was shaken overnight for complete extraction and spun down at 13,200 RPM for 8 minutes. Supernatant containing KTpCIPB was diluted 4 -fold by addition of methanol and sample concentration was determined using HPLC with corresponding standards injected separately.

Waters HPLC system (Waters, Milford, MA) was used. It consisted of a 1525 Binary HPLC pump, 717 plus autosampler, and 2487 Dual dabsorbance detector. Waters Breeze ${ }^{\circledR}$ chromatography software was used to acquire and process data. No column was used. Chromatography software was used to acquire and process data. No column was used. Chromatography was performed using $100 \%$ acetonitrile as the mobile phase, at a flow rate of $1 \mathrm{~mL} / \mathrm{min}$ and UV detection was set at $232 \mathrm{~nm}$.

\subsubsection{General drug absorption loading into nanoparticles}

Drug was dissolved to desired concentrations in deionized water (DIW). Both the drug solution and a nanoparticle suspension (Known concentrations) were added consecutively into an aqueous phased so that 1) drug and nanoparticle reached desired concentrations and relative weight ratios and 2) finals suspension volume reached $4 \mathrm{~mL}$. Mixtures were shaken (Ika KS130) for different lengths of time at different temperatures.

After drug loading, mixtures were dialyzed in Spectra/por 7 dialysis bag (MWCO: 50,000, Spectrum) against 1 liter of DIW (replaced every hour during the first $3 \mathrm{~h}$ ) over $24 \mathrm{~h}$. Dialyzed suspensions were used for size, zeta potential, and drug loading determination.

\subsubsection{Drug loading determination}

This procedure is similar to KTpCIPB loading determination. Briefly, drug loaded nanoparticle samples were lyophilized and weighed into $2 \mathrm{~mL}$ polypropylene tubes. $0.1 \mathrm{~mL}$ methylene chloride was added to nanoparticles, followed by shaking for $10 \mathrm{~min}$, then addition of $1.5 \mathrm{~mL}$ methanol and shaking for over $12 \mathrm{~h}$. These suspensions were spun down at 13,200 RPM for $8 \mathrm{~min}$. Supernatant containing dissolved drug was diluted as appropriate using methanol. Drug concentration was analyzed using UV doxHCl concentration determination procedure above.

\subsubsection{Vinblastine sulfate uptake study}

Two types of nanoparticles were used for this study. 502H:PEG-PLA(7:1, wt/wt) mixed polymeric nanoparticles with or without incorporation of maximal amount of KTpCIPB in the organic phase formulated at total polymer concentration of $20 \mathrm{mg} / \mathrm{mL}$ in ethyl acetate. Different nanoparticle formulations were loaded with vinblastine sulfate (VS) using the incubation method with variations.

\begin{tabular}{lcc}
\hline Formulation & Drug uptake temperature $\left({ }^{\circ} \mathbf{C}\right)$ & KTpCIPB presence \\
\hline 1 & Room temp & No \\
2 & 37 & No \\
3 & 37 & Yes \\
\hline
\end{tabular}

The above table describes the three groups of nanoparticles incubated with vinblastine sulfate. Nanoparticles were mixed polymeric nanoparticles with $20 \mathrm{mg} / \mathrm{mL}$ polymeric concentration in ethyl acetate. For the incubation drug uptake procedure, $7.5 \mathrm{mg}$ of blank nanoparticles were mixed with $1.5 \mathrm{mg}$ of VS in a total of $4 \mathrm{~mL}$ DIW and incubated at different temperature, with shaking, for 30min. Drug loaded nanoparticles were dialyzed (spectra/por 7, MWCO: 50K) against $1 \mathrm{~L}$ of DIW (replaced every hour for the first $3 \mathrm{~h}$ ) over $24 \mathrm{~h}$. Nanoparticles were lyophilized and then weighed out. VS was extracted from nanoparticles by adding $0.1 \mathrm{~mL}$ methyelene chloride and $1.5 \mathrm{~mL}$ methanol to pre-weighed nanoparticles followed by shaking for $12 \mathrm{~h}$. The 
suspension was spun down and supernatant containing VS was injected into HPLC for concentration determination.

A Waters HPLC system used for doxHCl detection was also used for VS detection. No HPLC column was used and the mobile phase was methanol: $10 \mathrm{mM}$ sodium phosphate (60:40). Flow rate was $1 \mathrm{~mL} / \mathrm{min}$ and injection volume was $100 \mu \mathrm{L}$.UV detection was set at $270 \mathrm{~nm}$ and VS in the samples were analyzed using corresponding standards injected separately.

\subsubsection{Plasma drug extraction procedure}

Goat plasma was aliquoted into $36-3 \mathrm{~mL}$ portions and divided into 12 groups of 3 portions each. Into six of the groups (control groups), $10 \mathrm{mg} / \mathrm{mL}$ doxHCl solution was added to plasma to reach either $15 \mu \mathrm{g} / \mathrm{mL}$, $30 \mu \mathrm{g} / \mathrm{m}, 50 \mu \mathrm{g} / \mathrm{mL}, 100 \mu \mathrm{g} / \mathrm{mL}, 150 \mu \mathrm{g} / \mathrm{mL}$, or $200 \mu \mathrm{L}$ followed by addition of $25 \mu \mathrm{L}$ of deionized water (DIW). Into the remaining six groups, $10 \mathrm{mg} / \mathrm{mL}$ doxHCl was added to reach the above concentrations followed by addition of $25 \mu \mathrm{L}(0.95 \mathrm{mg})$ of blank PEGylated nanoparticle suspension.

The 12 groups were shaken at $37^{\circ} \mathrm{C}$ for 30 minutes. Six groups containing nanoparticles were first centrifuged at 21,000 RPM for 25 minutes to spin down the nanoparticles ; supernantant samples were transferred to labled tubes. 0.4. $\mathrm{mL}$ of plasma from each sample in each group was transferred into $15 \mathrm{~mL}$ polyparopylene tubes followed byu addition of $4 \mathrm{~mL}$ methanol; choloroform $(3: 1, \mathrm{v} / \mathrm{v})$,1 minute voretexing, and shaking for 10 minutes. Samples were then centrifuged at 12,000 RPM for 10 minutes and $2 \mathrm{~mL}$ of organic phase was transferred into $50 \mathrm{~mL}$ polypropylene tubes and dried.

Dried phase was reconstituted using $0.3 \mathrm{~mL}$ methanol (for $15-100 \mu \mathrm{g} / \mathrm{mL}$ doxHCl samples) or $0.6 \mathrm{~mL}$ methanol (for 150 and $200 \mu \mathrm{g} / \mathrm{mL}$ doxHCl samples) and drug concentration was analyzed using HPLC (with no column) with corresponding standards injected separately.

\subsubsection{Borate selection and properties}

\section{Results and Discussion}

Borate-based lipophilic anions have traditionally been utilized as exchangers in ion-selective electrodes (Ganjali, Norouzi et al, 2006; Gupta, Singh et al. 2008). For this reason, different properties for these anions, relevant for nanoparticle encxapsulation, either hjave not been studied or have not been published. For this project, two critical properties of borates were $\log \mathrm{P}$, or partition coefficient of the anions between hydrophobic and hydrophilic phases, and solubilities in different organic solvents. The former could give a rough estimate of compatibility of these anions with hydrophobic cores of nanoparticles while the latter may suggest solvents appropriate for nanoparticle formulation. For borate-based compounds in this study (Fig.3.2), $\log$ P values were obtained as estimates using ChemDraw program (Table 3.1) and their colubilities in different organic solvents were determined by incremental addition of organic solvents to each borate compound (Fig.3.2).

Borate anions demonstrated increasing $\log \mathrm{P}$ valuews (increasing hydrophobicity), which indicated good hydrophobicity and compatible with nanoparticle cores (Fig.3.2. Table 3.1.). With addition of halogen and carbon atoms to the aromatic rings of the borates, hydrophobicity increased dramatically and could suggest design patterns which may be helpful when synthesizing new biocompatible lipophilic anions more appropriate for human usage. There did not appear to be a correlation between increasing $\log \mathrm{P}$ values and solubility in organic solvents (Table 3.1, Fig. 3.3.). KTTB was not soluble in any organic solvents tested and was not studied. For polymeric nanoparticle formulation, the most commonly used organic solvents for preparation are methylene chloride, chloroform, and ethyl acetate. Because methylene chloride and chloroform dissolved the borates very poorly, ethyl acetate, which dissolved the borates up to $2.5 \mathrm{mg} / \mathrm{ml}$., was chosen as the organic solvent for nanoparticle preparation.

\subsubsection{DoxHCl extraction by borate molecules.}

Knowing that borates could dissolve in ethyl acetate, it was important to show that they could also extract a model hydrophilic drug, doxorubicin hydrochloride (doxhCl), form aqueous to the ethyl acetate phase, by itself, doxHCl had negligible solubility in ethyl acetate (Fig.3.4.A)and could be extracted, but with increasing amounts of borates in the ethyl acetate phase, doxHCl extraction improved significantly(Fig. 3.4B). quantitatively, dox $\mathrm{HCl}$ extraction was $93.4 \%$ for $\mathrm{NaTPB}, 97.0 \%$ for KTpCIPB, and $94.9 \%$ for KTBTFMPB at 1:1 molar ration of borate to drug (Fig.3.5). thisd suggested the extraction process was primarily due to electrostatic interactions between anionic borates and cationic drug molecules and demonstrated the potential for doxorubicin to enter hydrophobic cores of borate-loaded naoparticles.

Previous studies using NaTPB as ion exchangers showed leaching of the electrostatically bound material, suggesting ;it was not the best borate for future studies; while KTBTFMPB was 20 times more expensive than the other borate anions. For the above reasons, KTpCIPB was chosen as the model lipophilic anion for nanoparticle studies. 


\subsubsection{Nanoparticle formulation with encapsulated KTpCIPB}

KTpCIPB has not been associated with nanoparticle formulations in previous publications. For this study, an oil-in-water $(\mathrm{O} / \mathrm{W})$ emulsion method was used to formulate $\mathrm{KTpCIPB}$ - loaded polymeric nanoparticles (Fig.3.7). Because both the polymer chosen, PLGA and the borate-based anion, KTpCIPB, could dissolve in ethyl acetate, the procedure was simple as ethyl acetate with dissolved KTpCIPB and PLGA was emulsified into an outer aqueous phase to formulate the particles. To validate successful nanoparticle formation, particle sizing was done to determine the number and size of nanoparticle polulation(s) and scanning electron mircroscopy(SEM) was done to observe the morphology of the nanoparticles. The results were positive as particle size (not shown here) showed a unimodal (one population or peak) distribution with an average size below $200 \mathrm{~nm}$ depending on the initial amounts of KTpCIPB added and SEM images (Fig.3.8) showed spherical nanoparticles with similar sizes range of between $100-250 \mathrm{~nm}$.

\subsubsection{KTpCIPB presence of quantification in nanoparticles}

Despite a monomodal size distyribution, direct evidence of DTpCIPB incorporation into the naoparticles was still needed and two other experiments were done to confirm its incorporation. First, the O/W emulsion procedure was repeated except that only KTpCIPB, and no polymer, was dissolved in the ethyl acetate phase. The resulting cmulsion was clear, filtered through the stir cell membrane filter system (used for washing nanoparticles) rapidly unlike nanoparticles suspensions with KTpCIPB encapsulated, which did not filter through at all. This demonstrated that any KTpCIPB not encapsulated into nanopartciles would be removed from the naoparticle solution during the washing procedure and any changes in particle size or drug loading for KTpCIPB - loaded versus KTpCIPB-free nanoparticles was due to KTpCIPB actually associated with nanoparticles. Second, and more importantly, V was extracted from lyophilized nanoparticles with 0 or $10.7 \%$ theoretical KTpCIPB loading (Table 3.2). HPLC analysis of extracted supernatant from KTpCIPB-loaded nanoparticles showed a large peak which was not present for extracted supernatant of KTpCIPB-free nanoparticles. Another important result from this HPLC experiment was that incubation of KTpCIPB-loaded nanoparticles in different solutions (PBS or DIW) for $1 \mathrm{~h}$ or less did not appear to cause KTpCIPB loss as calculated percent loading results for non-incubated and incubated nanoparticles were similar. In addition, it was observed that only $40 \%$ of the initial KTpCIPB added was actually encapsulated (Table 3.2.), which suggested an upper limit on how much KTpCIPB could actually be incorporated. The above results clearly demonstrated encapsulation of KTpCIPB into the nanoparticles although more than half of the KTpCIPB was lost during formulation. This experiment, however, did not show the location of these molecules in nanoparticles (core or surface or both)

\subsubsection{Optimization of incubating drug loading}

Under optimal conditions, absorption drug loading, where drug is incubated with and extracted into pre-formulated nanoparticles, may prove to be a versatile strategy to improve drug loading and other properties using PLGA-based nanoparticles. To optimize these conditions, various factors were analyzed including 1) PLGA acid number, 2) increasing KTpCIPB loading, 3) impact of temperature, 4) different weight ratios of nanoparticles to drug in the incubation media, and 5) impact of PEGylation of nanoparticles.

\subsubsection{Polymer(PLGA) selection}

Many different polymers have been specially synthesized for the purpose of absorption drug loading, however, PLGA, the most readily available polymer approved by the FDA for clinical use, has not been systemically studied for absorption loading/uptake purposes much less in combination with KTpCIPB. For this study, three different PLGA 50/50 polymers were selected (Table 3.3) to formulate blank nanoparticles (no KTpCIPB added) to isolate the property most important for choosing the best PLGA. Of the three, 502H had the smallest molecular weight and viscosity, indicating that nanoparticles formulated using this polymer should be smaller than particles formulated using the more viscous polymers. More importantly, the acid numbers of the polymers were quite different. Resomer 503 was end-capped with acid number of $<1$, meaning the majority of carboxylic acid groups at the end of each polymer strand were "capped" by an alkyl group to form alkyl esters. Because carboxylic acid groups could contribute negative charges for electrostatic attraction to cationic drugs, the polymer with the highest acid number, resomer $502 \mathrm{H}$, was expected to produce nanoparticles with the highest drug loading. Results confirmed this hypothesis as $502 \mathrm{H}$ nanoparticles (ethyl acetate organic phase) showed substantially higher extraction efficiency and drug loading (Fig.3.10A) relative to nanoparticles made with other polymers, reaching $1.44 \%$ drug loading and $44.6 \%$ extraction efficiency. In comparison, nanoparticles formulated using resomer 503, the end-capped. Another interesting result from this figure was the resomer $503 \mathrm{H}$ nanoparticles made with ethyl acetate organic phase which were smaller than ones made with methylene chloride, had twice the drug loading and significantly improved encapsulation efficiency. 
The results confirmed that acid number was important for enhancing drug loading and encapsulation efficiency and polymers with highest acid number should be selected while smaller particles, with greater surface area-tovolume ratio and potentially higher polymer density, was also important for improving adsorption drug loading.

\subsubsection{KTpCIPB incorporation}

Even though anionic polymers or copolymers have been synthesized in the past to increase electrostatic interactions with cationic drugs, small molecule lipophilic anions such as KTpCIPB have not been studied for this purpose and optimal amount of KTpCIPB for nanoparticle incorporation was uncertain.

As the data showed, increasing KTpCIPB incorporation increased extraction efficiency (measure of how much drug initially present in the incubation media was taken up by the particles) from $48 \%$ to $75 \%$ at the highest KTpCIPB loading, and drug loading from 3.0\% without KTpCIPB addition to $4.8 \%$ at maximal KTpCIPB loading (Fig $3.11 \mathrm{~A}$ ). These results clearly demonstrated the benefits of lipophilic anions and also suggested that additional KTpCIPB incorporation would continue to increase drug loading; however, since KTpCIPB incorporation in this study was limited by its solubility in ethyl acetate, further improvements in drug uptake was unlikely.

Previous results had shown approximately $40 \%$ encapsulation efficiency of KTpCIPB into nanoparticles (Tabel 3.2). Based on this result, when maximal KTpCIPB amount was loaded into nanoparticles (4.8 mg KTpCIPB for $80 \mathrm{mg}$ polymer nanoparticles), only $2.16 \mathrm{mg}$ of KTpCIPB was encapsulated in $80 \mathrm{mg}$ of polymer nanoparticles. Since doxHCl loading was improved by $1.8 \%$ (3\% to $4.8 \%$ as stated above) due to maximal KTpCIPB addition, this meant an additional $1.44 \mathrm{mg}$ of doxHCl was loaded for every $80 \mathrm{mg}$ of polymer in nanoparticles due to KTpCIPB and $1.44 \mathrm{mg}$ doxHCl meant a KTpCIPB: doxHCl molar ratio of 1.75:1, which suggested not all the encapsulated $\mathrm{V}$ was paired with a drug molecule and anionic charges were still available. However, KTpCIPB may be buried deep within the nanoparticles, precluding drug access. Furthermore, the results did not clearly indicate if hydrophobic interactions (pi-pi stacking) played a factor in the increased drug loading.

Another interesting observation was that in the presence of increasing KTpCIPB, nanoparticle size decreased dramatically from $182.5 \mathrm{~nm}$ to $117.1 \mathrm{~nm}$ (Fig.3.11B). One hypothesis for this was that increasing KTpCIPB may lead to more drug loading and more hyudrophobic drug- KTpCIPB ion pair formation, especially on particle surface. These hydrophobic ion pairs could drive water away from, or even out of, nanoparticles and lead to a smaller hydrodynamic radius recognized by the particle sizing instrument.

\subsubsection{Temperature effect on adsorption uptake}

With increasing KTpCIPB loading into 502H nanoparticles, doxHCl loading and extraction efficiency could be improved significantly, as results showed. Even though the previous KTpCIPB uptake studies were done at $37^{\circ} \mathrm{C}$, the physiological temperature, it was important to discern the effects of temperature on drug loading and extraction efficiency. Comparison of drug adsorption loading for KTpCIPB-loaded nanoparticles at room and physiuological temperatures showed a very interesting trend wherfe all other conditions being equal, higher temperature generated approximately 30\% higher drug loading at all KTpCIPB loadings (Fig.3.12A) aswell as similar increases in extraction efficiency (data not shown). For example, drug loading for nanoparticles with no KTpCIPB incorporated imporved form 1.4\% to 3.0\% while nanoparticles with maximized KTpCIPB loading improved form $3.7 \%$ to $4.8 \%$. These data demonstrated temperature-dependent drug loading improvement was actually due to the polymer used $(502 \mathrm{H})$ themselves rather than because of KTpCIPB. A hypothesis for this increase focused on polymer rearrangement in nanoparticles. Polymeric nanoparticle could be envisioned as a ball of polymer strands with each strand having random motion. Hydration of nanoparticles and residence in an environment where temperature was above the glass transition temperature $\left(T_{g}\right)$ for the polymer were known to significantly increase the polymer strand motion and lead to rearrangement within a nanoparticles. For PLGA, an experimentally determined $\mathrm{T}_{\mathrm{g}}$ value was approximately $31^{\circ} \mathrm{C}$ (Blasi, D'Souza et al. 2005). Which was in between the room temperature and physiological temperatures studied and led to the conclusion that in this case, increasing polymer rearrangement due to temperature increase led to increase drug molecule partitioning into the polymer phase and ion-paid formation with polymer carboxylic acids and KTpCIPB. This phenomenon was also cited in a previous article from Kang and colleagues (Kang and Schwendeman 2003), where partitioning of small hydrophobic probes into polymer films increased with increasing temperature.

\subsubsection{Effect of drugmanoparticle ratio on drug uptake}

For absorption loading, relative amounts of nanoparticle and drug in solution could be important due to a passive drug concentration gradient. However, it was uncertain how much KTpCIPB presence could impact this and at what drug; nanoparticle weight ratio further increases in drug amount would make no difference. 
For this study, fixed amount of KTpCIPB-loaded nanoparticles were incubated with increasing amounts of dox $\mathrm{HCl}$ in solution up to $1.5 \mathrm{mg}$. however, at the higherst ratio of $1.5 \mathrm{mg}: 15 \mathrm{mg}$ (drug:nanoparticle), nanoparticle aggregation and sedimentation occurred during incubation. Between additions of $0.25 \mathrm{mg}$ to $1 \mathrm{mg} \mathrm{doxHCl}$, though, results (Fig.3.13A) showed almost complete doxHCl extraction efficiency (95\% or above) with $0.25 \mathrm{mg}$ or $0.5 \mathrm{mg}$ doxHCl in solution and $75 \%$ when $1 \mathrm{mg}$ doxhCl was present. Drug loading also increased from $1.6 \%$ (with $0.25 \mathrm{mg}$ dox $\mathrm{HCl}$ added) to $4.8 \%$ (with $1 \mathrm{mg}$ dix $\mathrm{HCl}$ added): this was logical given the higher drug concentration gradient explained before and higher driving force for partitioning into nanoparticles.

Nanoparticle zeta potential was more positive with drug adsorption loading (changing form $-35 \mathrm{mV}$ to -26 ,V). which was reasonable considering surface negative charges were being neutralized; however, there was no significant change in zeta potential nor particle size with increasing amounts of drug $(0.25 \mathrm{mg}-1 \mathrm{mg})$ in the incubation media (Fig. 3.13B). this suggested that additional loaded drug were below the top layer of nanoparticles, supporting the polymer strand rearrangement theory in the previous temperature study.

Drug loading versus amount of doxHCl added curve increased in an almost linear fashion without reaching a plateau, suggesting that not all potential drug binding sites were occupied on nanoparticles and higher loading could be achieved if nanoparticle aggregation problem could be resolved.

\subsubsection{PEGulated nanoparticles.}

Traditionally, it was known that hydrophilic moiety (polymer) could be grafted onto nanoparticle surface to form a hydrophilic surface layer that repels protein in adsorption onto nanoparticle suface as well as reduce potential aggregation between particles. A commonly used hydrophiclic polymer for this purpose is poly(ethylene glycol) (PEG)(Heald, Stolnik et al, 2002), a biodegradable polymer used in commercial products. For formulation purposes, PEG was usually incorporated through direct conjugation to a hydrophobic polymer, such as poly (lactic acid) (PLA), to form a diblock copolymer, which may be used by itself or in combination with other polymers to form nanoparticles. As copolymer nanoparticles form, PLGA or PLA segment intertwine to form a hydrophobic nanoparticle core while the hydrophilic PEG segments extend out to form a hydrophilic shell (Fig.3.14).

\subsection{NMR characterization}

Here, PEGylated nanoparticles were made from a mixture of resomer 502H and synthesized PEG-PLA although the total polymer concentration for these PEGylated nanoparticles was the same as regular nanoparticles made with only resomer 502H. NMR spectrum of the copolymer (Fig.3.6A) matched those in previously published papers (Olivier, Huertas et al. 2002) and based on comparison of the various peaks obtained, calculated molecular weights of between 19,408-22,689 Da were obtained which was similar to the theoretical value of $25,000 \mathrm{Da}$ found based on the weight ratio of L-lactide and $\mathrm{mPEG}-\mathrm{NH}_{2}$ used to synthesize the copolymer(Fig.3.6B).

\subsection{XPS characterization}

X-ray photoelectron spectroscopy (XPS) has been used extensively for surface analysis to various polymeric materials. Using this technique, binding energies of electrons associated with different atoms in the top 2-10 nm of a surface can be measured to collect elemental and chemical information (Scholes, Coombes et al. 1999; Dong and Feng 2004). Here, XPS was utilized to analyze six nanoparticle samples including 1) blank PLGA (502H), 2) blank PEG-PLA, 3) blank PLGA-PLA (7:1, wt/wt), nanoparticles, all without KTpCIPB addition; as well as 4) blank PLGA (502H), 5)blank PLGA:PEG-PLA (7:1,wt/wt), and 6) doxorubicin-loaded PLGA:PEG-PLA(7:1,wt/wt), all 3 of which had maximal KTpCIPB incorporation. These samples were investigated for chemical composition as well as the presence of dox $\mathrm{HCl}$ and PEG polymers on nanoparticle surface.

Survey scans of the nanoparticle samples (Fig,3.15) were done to detect carbon, oxygen and nitrogen atoms on nanoparticles surface and their percent composition. Of the materials present in nanoparticles, only the drug, doxorubicin hydrochloride (doxHCl), contained nitrogen atoms in its structure, thus presence of nitrogen peak at around $400 \mathrm{eV}$ in the survey scans would indicate drug accumulation on nanoparticle surface. However, all six survey scans were similar in shape and showed visible $\mathrm{C}_{\mathrm{ls}}$ and $\mathrm{O}_{\mathrm{ls}}$ peaks, while no nitrogen peaks were present. Quantification results further confirmed this as all six samples, with the exception of blank KTpCIPBloaded PLGA $(502 \mathrm{H})$ nanoparticles, had zero nitrogen content (Table 3.4.) .Because the detection limit of this instrument is $0.1 \%$ though, even the $0.1 \%$ nitrogen composition for blank KTpCIPB-loaded PLGA nanoparticles was negligible. Upon analysis, this result was expected because based on the chemical formula of dox $\mathrm{HCl}, \mathrm{C}_{27} \mathrm{H}_{29} \mathrm{NO}{ }_{11} \mathrm{Cl}$, nitrogen has a theoretical percent atomic composition of $2.5 \%$ within doxorubicin; and when embedded into or onto nanoparticles, surrounded by numerous other carbon and oxygen atoms of polymers, this was easily reducible to less than $0.1 \%$. from survey scan results, it was hard to draw conclusions regarding dox $\mathrm{HCl}$ localization within nanoparticles. 
A surprising result from the survey spectra was that the percent atomic composition of carbon was similar to that of oxygen (Table 3.4), different from a previous study showing a 2:1 atomic ratio of carbon:oxygen (Pourcelle, Devouage et al, 2007), and different from predicted theoretical values of 2:1 for PEG, 2:5:2 for PLGA and 3:2 for PLA, all based on their respective chemical formulas. Considering that even the controls for this study, blank PLGA and blank PEG-PLA nanoparticles, both had low carbon:oxygen atomic ratios, this result could be due to a problem with instrument calibration where theintensity reading for the carbon peak was not fully optimized. Form the atomic composition of carbon(Table 3.4.), it was observed that carbon composition for blank PEG-PLA nanoparticles was 5\% higher than blank PLGA nanoparticles (43.6\% vs. $48.6 \%$ ), which was expected given the higher theoretical carbon: oxygen ratio for PEG. Based on this, it was also not surprising to see blank PLGA:PEG-PLA nanoparticles with a percent carbon composition in between that of PLGA and PEG-PLA nanoparticles and this result was direct evidence of the presence of PEG strands on naoparticle surfaces.

Results from Table 3.4 also showed that percent carbon composition for blank PLGA:PEG:PLA and blank PLGA/ KTpCIPB nanoparticles were similar (45.8\% vs. 45.4\%). Because there were no PEG-PLA in the KTpCIPB loaded nanoparticles, a potential explanation for the increased carbon composition was due to KTpCIPB, which has an amphiphilic structure (Fig.3.2) that promoted localization on nanoparticle surfaces (similar to detergents) as well as number of benzene rings in its structure which contribute to percent carbon composition. However, another potential cause could be polyvinyl alcohol (PVA), a surfactant added to all six formulations that could localize to the surface of nanoparticles to stabilize them. Like PEG, PVA also has 2:1 carbon :oxygen atomic ratio and the combination of PVA+PEG-PLA and PVA+ KTpCIPB on naoparticle surfaces could lead to similar carbon:oxygen ratios if enough PVA was present.

Lastly, results showed percent carbon composition for blank PLGA:PEG-PLa/ KTpCIPB and PLGA:PEG-PLA/ KTpCIPB/dox nanoparticles were significantly higher than the other four batches (Table 3.4). This result was surprising considering the magnitude of the increase relative to the control blank PLGA nanoparticles $(51.2-52.1 \%$ to $43.6 \%$, 7-9\% increase ). Blank PLGA:PEG-PLA and blank PLGA/ KTpCIPB nanoparticles each had approximately $2 \%$ higher carbon composition relative to blank PLGA nanoparticles, which meant a combined increase of $4 \%$, or around $47.6 \%$ theoretical carbon composition for nanoparticles with both PEG-PLA and KTpCIPB added. However, actual carbon compositions were $51.2 \%$ and $52.1 \%$, or between 3-5\% higher than expected, for PLGA nanoparticles containg PEG-PLA and KTpCIPB. This phenomenon could be due to synergistic actions between PEG-PLA and KTpCIPB, which multiplied rather than simply added the contributions of the components, when both components were present in nanoparticles. For examples, by itself, PEG-PLA was miscible with PLGA and the combination of the two on nanoparticle surface led to a lower carbon:oxygen ratio than if only PEG-PLA was present on the surface. However, the presence of KTpCIPB on nanopartciles appeared to reduce this miscibility and caused PEG-PLA to be pushed to the surface of nanoparticles, away from the PLGA core. Thus the combination of PEG-PLA with KTpCIPB, with its many benzene rings and carbon atoms, firmed a thick enough layer on nanoparticle surfaces to increase percent carbon compositing readings.

It was found that blank PLGA:PEG-PLA nanoparticle had slightly more hydrocarbons and PEG carbons relative to blank PLGA nanopartcles (less than 1 percent each, Table 3.5). Based on the fact that instrument detection limit was $0.1 \%$ and PEG-PLA only constituted $12.5 \%$ (wt/wt) of each nanoparticle, this again showed that some PEG was present on the surface layer. This result also matched previous percent carbon compositions results (Table 3.4.), where blank PLGA-PEG-PLA nanoparticles had approximately $2 \%$ higher carbon composition relative to blank PLGA nanoparticle due to PEG presence.

Earlier, it was proposed that when both KTpCIPB and PEG-PLA were incorporated into nanoparticles, PEG-PLA would have reduced miscibility with PLGA and PEG-PLA, along with KTpCIPB, could form a surface layer that shielded the PLGA core. This was again confirmed through the carbon decomposition results (Table 3.5). For OKGALOEG-PLA/ KTpCIPB nanoparticles with and without doxHCl, both hydrocarbons and PEG carbon percentages were much higher than polyester and carboxyl carbons, both of which were normally indicative of PLGA polymers. Furthermore, it was very interesting to see the PEG carbon percentages for these two nanoparticle formulations $(32,79 \%$ abd $34.20 \%)$ to be much higher than even blank PEG-PLA nanoparticles (18.04\%)while polyester and carboxyl carbon percentages were much lower. This result indicated that the combination of KTpCIPB with $12.5 \%$ weight percent PEG-PLA in PLGA nanoparticles may be a thicker layer than the PEG layer of pure PEG-PLA nanoparticles.

Lastly, an important observation from Table 3.5 was that PLGA:PEG-PLA/ KTpCIPB nanoparticles, with doxorubicin loaded, contained $42.99 \%$ hydrocarbons, while the batch with no doxorubicin had $40.47 \%$ hydrocarbons. Because the structure of doxorubicin (Fig.3.1.) contained many fused rings with numerous C-C, $\mathrm{C}-\mathrm{H}$ (hydrocarbon ) carbons, the $2.52 \%$ increase in hydrocarbon reading would be attributed to the presence of doxorubicin. Even though it was nearly impossible to calculate the number of doxorubicin molecules 
representing this $2.52 \%$ increase, qualitatively, it was safe to say that part of the doxorubicin loaded into these nanoparticles via incubation loading was in the surface layer.

\subsection{SEM characterization}

SEM images showed PEGylated nanoparticles, like regular ones, were spherical with size range of between 100-200 nm (Fig. 3.18). to study potential improvements of PEGylated nanoparticles, increasing amounts of doxHCl were again incubated with fixed amount of PEGylated nanoparticles upto $2 \mathrm{mg}$ doxHCl. Because results at $2 \mathrm{mg}$ dox $\mathrm{HCl}$ addition were almost the same as those at $1.5 \mathrm{mg}$ doxHCl addition, these results were not shown. With increasing dox $\mathrm{HCl}$ addition to PEGylated nanoparticles, no visible aggregation occurred: suggesting surface PEG strands were effective for repelling other particles. Despite no visible aggregation, particle sizing data of PEGylated nanoparticles (Fig 3.17C), after incubation with $1 \mathrm{mg}$ and $1.5 \mathrm{mg} \mathrm{doxHCl}$, showed increased particle size. This amy be explained by transient particle-particle interactions where PEG strands momentarily intertwined and then came apart or where nanopartciles surfaces without PEG coverage interact to form transient complexes. Overall, particle size still remained below $350 \mathrm{~nm}$, which was in line with or below many previously reported particles diameters.

\subsection{Drug loading}

Dox $\mathrm{HCl}$ extraction from solution, again, decreased with increasing drug in the incubation media while drug load increased due to higher doxHCl concentration gradient(Fig.3.17A). Extraction efficiency of PEGylated nanoparticles was slightly below that for regular nanoparticles, and with $1.5 \mathrm{mg}$ dox $\mathrm{HCl}$ in the incubation media, extraction efficiency by PEGylated nanoparticles was still as high as $65 \%$. The slightly lower extraction efficiency may be attributed to the outer shell of PRG strands that may repel a small percentage of doxHCl molecules in solution and slow their migration into the nanoparticle. Despite the substitution of PEGPLA for a portion of resomer 502H, which reduced the number of carboxylic acids available for drug binding, drug loading values for regular and PEGylated nanoparticles were similar across the different drug;nanoparticle ratios. Infact, by preventing aggregation, PEGylated nanoparticles with maximal KTpCIPB loading achieved upto $6 \%$ drug loading (Fig.3.17B), which was much higher than most published drug loading values.

\subsubsection{Vinblastine sulfate uptake}

Vinblastine sulfate (VS), like doxorubicin hydrochloride, was commonly used for cancer treatment. As a small molecule drug, it has molecular weight of 810,97 daltons with a number of fused rings in its structure (Fig.3.19) that could be beneficial for hydrophobic interaction, much like doxHCl. In terms of physicochemical properties, VS has moderate solubility in aqueous medium (greater than $10 \mathrm{mg} / \mathrm{mL}$ ) and a reported pKa of 7.4(Gaertner, Murray et al. 1998), which meant that under physiological conditions, around 50\% of VS molecules should have a net positive charge. Because its intrinsic properties matched the type of drug whose loading we would like to improve using the specially formulated KTpCIPB-loaded PEGylated nanoparticles, vinblastine sulfate was used as an additional model drug to demonstrate the potential utility of these nanoparticles toward this category of drug.

Several formulations were studied using either KTpCIPB-free or KTpCIPB-loaded PEGylated nanoparticles to further demonstrate improvements due to KTpCIPB. PEGylated nanoparticles were loaded with vinblastine sulfate at either room temperature or $37^{\circ} \mathrm{C}$ to see if the temperature-induced drug loading increase seen before also applied. KTpCIPB-loaded PEGylated nanoparticles with no VS loaded was used as a control.

Results showed that as drug loading temperature increased from room temperature to $37^{\circ} \mathrm{C}$, actual drug loading improved from $3.77 \%$ to $5.98 \%$ while encapsulation efficiency improved from $22.6 \%$ to $35.9 \%$ (Table 3.6). This improvement again showed that the temperature effect on drug loading was dependent on the nanoparticles or polymer used and this system was effective for both ; doxHCl and VS. With KTpCIPB incorporation into the nanoparticles, drug loading increased from 5.98\% to 8,98\% and encapsulation efficiency improved from $35.9 \%$ to $53.9 \%$ 9Table 3.6). This was significant as almost half of the drug initially present in the incubation medium was taken up by the nanoparticles and further showed that KTpCIPB presence significantly enhanced drug loading. It was also intresting to see that vinblastine sulfate loading into these nanoparticles was higher than for dox $\mathrm{HCl}$ and this could be due to the additional fused rings in its structure that provide imporved stability in the hydrophobic core of nanoparticles. Alternatively, this loading difference relative to dox $\mathrm{HCl}$ may be due to the higher molecular weight of VS.

The above results with VS provided evidence that drug loading improvements using KTpCIPB-loaded PEGylated nanoparticles were not exclusive to doxorubicin hydrocholoride and it would be interesting to know if these particles and drug loading systems could be applied to other durgs. 


\subsubsection{Charge quantification calculations}

Previous studies demonstrated significant increase in doxHCl and VS drug loading when the polymer, $502 \mathrm{H}$, and $\mathrm{KTpCIPB}$ were used to formulate the ion complexing nanoparticles. Assuming that all available charges of 502H polymer and KTpCIPB could be used for ion pairing with drug molecules on a charge-forcharge basis, it would be interesting to calculate the theoretical drug loading in these nanoparticles.

First, the effect of $502 \mathrm{H}$ polymers on doxHCl loading was calculated. $502 \mathrm{H}$ polymers have an acid number of minimum $6 \mathrm{mg} \mathrm{KOH/gram} \mathrm{polymer} \mathrm{according} \mathrm{to} \mathrm{the} \mathrm{company} \mathrm{(Boehringer} \mathrm{Ingelheim)website.} \mathrm{This}$ meant that since $\mathrm{HOH}$ has a molecular weight of $56.1 \mathrm{~g} / \mathrm{mol}$, approximately $17.82 \mu \mathrm{mol}$ of $\mathrm{KOH}$ may be neutralized by protons contributed by carboxylic acids in one grams of this polymer, which was also the same as saying that $17.82 \mu \mathrm{mol}$ of doxorubicin could be neutralized by one gram this polymer. Because $80 \mathrm{mg}$ of $502 \mathrm{H}$ was used to make each batch of nanoparticles, this meant $1.426 \mu$ or $0.827 \mathrm{mg}$ (doxHCl MW:580 g/mol) of doxorubicin could be loaded into these nanoparticles by ion pairing. Finally, $0.827 \mathrm{mg}$ of dox $\mathrm{HCl}$ in $80 \mathrm{mg}$ of $502 \mathrm{H}$ nanoparticles would be equivalent to $1.02 \%$ drug loading. This theoretical drug loading was lower than the actual loading observed in the temperature-dependent drug uptake study(Fig.3.12A), where drug loadings in $\mathrm{KTpCIPB}$-free $502 \mathrm{H}$ nanoparticles were $1.4 \%$ and $3 \%$, at room temperature and $37^{\circ} \mathrm{C}$, respectively. The increase in the actual drug loading could be due to hydrolysis of 502Hduring nanoparticle formulation which generated additional free acids in the polymer; although a more likely explanation was that the acid number reported by the manufacturer was only a minimum, and the actual acid number, was approximately 11.5(determined by standard titration with potassium hydroxide by Dr.Ying Zhang), or twice the reported value. This meant the theoretical drug loading should have been $2.04 \%$, which was closer to the actual values of $1.4 \%$ and $3 \%$.

Because only $2 \mathrm{~mL}$ of ethyl acetate was used during nanoparticle formulation and KTpCIPB had a solubility of $2.4 \mathrm{mg} / \mathrm{mL}$ in ethyl acetate, the maximal amount of KTpCIPB that was theoretically added into each batch of nanoparticles was $4.8 \mathrm{mg}$. Since a previous KTpCIPB extraction study found that encapsulation efficiency of KTpCIPB in nanoparticles was around $40 \%$ (Table 3.2), this meant that $1.92 \mathrm{mg}$ of KTpCIPB was actually encapsulated into each batch of nanoparticles. Assuming that each molecule of KTpCIPB could ion pair with amolecule of doxHCl and that molecular weights of $\mathrm{KTpCIPB}$ and doxHCl are $496 \mathrm{~g} / \mathrm{mol}$ and $580 \mathrm{~g} / \mathrm{mol}$, respectively, this meant $1.92 \mathrm{mg}$ of $\mathrm{KTpCIPB}$ can bind to $2.25 \mathrm{mg}$ of doxHCl. If $80 \mathrm{mg}$ of $502 \mathrm{H}$ was used to formulate nanoparticles, this would have been equivalent to $2.67 \%$ increase in drug loading due to KTpCIPB. Lokking at actual result, there was an increase in drug loading from 3\% to $4.8 \%$ between KTpCIPB-free nanoparticles and nanoparticles with maximal KTpCIPB incorporation (Fig.3.12A), which meant that presence of KTpCIPB led to $1.8 \%$ increase in loading. Comparing the actual increase of $1.8 \%$ and theoretical increase of $2.67 \%$ this meant that $2 / 3$ of available KTpCIPB were ion paired, which was substantial.

Lastly, it was observed that vinblastine sulfate loading into KTpCIPB-free nanoparticles were $3.77 \%$ and $5.98 \%$ at room temperature and $37^{\circ} \mathrm{C}$, respectively (Table 3.6) .Since $80 \mathrm{mg}$ of $502 \mathrm{H}$ were used to make each batch of nanoparticles, based on reported acid number for $502 \mathrm{H}$, this meant $1,426 \mu$ mol or $1.16 \mathrm{mg}$ (vinblastine $\mathrm{MW}: 811 \mathrm{~g} / \mathrm{mol}$ ) of vinblastine could be loaded into these nanoparticles by ion pairing. $1.16 \mathrm{mg}$ of vinblasine in $80 \mathrm{mg}$ of $502 \mathrm{H}$ would have been equivalent to $1.43 \%$ theoretical drug loading (charge basis). However, since the measured acid number was almost twice the reported value, this meant the theoretical drug loading should have been almost $2.9 \% /$ This theoretical drug loading almost matched drug loading at room temperature, $3.77 \%$, although it still fell short of the $5.98 \%$ achieved at $37^{\circ} \mathrm{C}$. one potential reason for the significantly higher drug loading relative to the theoretical value may be due to the structure of vinblastine, which contained more alkyl rings that may promote compatibility with the hydrophobic core of nanoparticles. In addition, if we again assume that $1.92 \mathrm{mg}$ of KTpCIPB was actually encapsulated into each batch of nanoparticles and that KTpCIPB and vinblastine sulfate have molecular weights of $496 \mathrm{~g} / \mathrm{mol}$ and $811 \mathrm{~g} / \mathrm{mol}$, respectively, this meant $1.92 \mathrm{mg}$ of KTpCIPB could bind $3.14 \mathrm{mg}$ of vinblastine sulfate. Since $80 \mathrm{mg}$ of $502 \mathrm{H}$ was used to formulate these nanoparticles, this meant the presence of KTpCIPB led to an increase in theoretical loading of $3.7 \%$. Because drug loading actually increased from $5.98 \%$ to $8.98 \%$ with incorporation of maximal amount of KTpCIPB (Table 3.6), the 3\% actual increase in drug loading was close to the theoretical improvement of $3.7 \%$ and indicated that approximately $80 \%$ of available KTpCIPB was bound to vinblastine molecules.

\subsubsection{Plasma drug uptake study}

Previous in vitro results demonstrated PEGylated nanoparticles in this study could effectively take up the model drugs, doxorubicin hydrochloride and vinblastine sulfate, after optimization. Ultimately, it was hoped that the successful in vitro results could translate into equivalent in vivo date;however, before conducting lenthy and difficult in vivo studies, a proof of concept should be done under simulated physiological conditions, or in plasma, which to the best of our knowledge, has not been done before. In this study, plasma suspensions containing different concentrations of dissolved dox $\mathrm{HCl}$ (from a normal therapeutic dose up to four times the 
therapeutic dose) were incubated with or without blank PEGylated nanoparticles and free drug concentration after incubation were determined,

To choose the proper conditions for this study, two things had to be calculated, one was an appropriate nanoparticle concentration in plasma, while the other was the normal dtherapeutic concentration of doxHCl in plasma (assuming 70\% plasma protein drug binding). Doxil ${ }^{\circledR}$, a nanoparticulate liposome containing doxHCl, was widely used clinically for cancer treatment, so is nanoparticle concentration per dose was used for this study. Normally, around 1 gram of liposomes was administered per dose and since an average person has around 3 liters of plasma, this calculated to a dose of $1 \mathrm{mg}$ nanoparticles $/ 3 \mathrm{~mL}$ of plasma $(\mathbf{1} \mathbf{m g} / \mathbf{3 m L})$. For doxHCl dose calculation, the normal dose of Adriamycin ${ }^{\circledR}$ i.e., $150 \mathrm{mg}\left(60-75 \mathrm{mg} / \mathrm{m}^{2}\right.$ while an average 6 feet human was around $2 \mathrm{~m}^{2}$ ) was used. Because the human body has around 3 liters of plasma, this translated to $50 \mu \mathrm{g} / \mathrm{mL} \mathrm{of}$ total dox $\mathrm{HCl}$ concentration in plasma. Since doxHCl binding by plasma protein was $70 \%$, free doxHCl concentration in plasma was $15 \mu \mathrm{g} / \mathrm{mL}$. in addition to these two theoretical drug concentrations, a concentration in between these $(30 \mu \mathrm{g} / \mathrm{mL})$ as well as potentially toxic doses of $2 \mathrm{X}, 3 \mathrm{X}$ and $4 \mathrm{X}$ the normal therapeutic concentration of $50 \mu \mathrm{g} / \mathrm{mL}$ were tested.

Plasma doxHCl concentrations, after drug extraction in the presence or absence of PEGylated nanoparticles, were measured while initial drug concentration were used to denote each formulation. Final free dox $\mathrm{HCl}$ concentration, without addition of nanoparticles, were smaller than the theortical drug concentrations due to plasma perotein drug binding. Calculations showed that drug binding was consistently around 60\% (not shown) while reported human plasma protein dox $\mathrm{HCl}$ binding was around $70 \%$ although values of between $50 \%$ and $70 \%$ have also been observed.

For the tested doxHCl concentrations near its normal therapeutic concentration $(15-50 \mu \mathrm{g} / \mathrm{mL})($ Table $3.7)$, the effect of nanoparticles were relatively insignificant as the percent drug concentration reduction was between $7.7 \%-9.44 \%$, which only translated to actual reduction of between $0.5-1.8 \mu \mathrm{g} / \mathrm{mL}$. Nanoparticle drug loading here was only between $0.18-0.6 \%$, which was insignificant considering these PEGylated could have a theoretical drug loading of up to 6-7\%. Between 100 and $150 \mu \mathrm{g} / \mathrm{mL}(2 X$ or $3 X$ the normal dose of $50 \mu \mathrm{g} / \mathrm{mL}$ ), the percent drug concentration reduction was $6.96 \%$ and $8.4 \%$, respectively, which was comparable to those at normal drug dose, but drug loading improved slightly to $0.9 \%$ and $1.66 \%$, showing that more drug molecules were starting to diffuse into the nanoparticles. At $200 \mu \mathrm{g} / \mathrm{mL}$ initial doxHCl concentration, absolute drug concentration reduction suddenly jumped to more than $13 \mu \mathrm{g} / \mathrm{mL}$, or $15.71 \%$. More importantly, drug loading of the nanoparticles increased to $4.35 \%$, an almost 3-fold increase compared with the study done at $150 \mu \mathrm{g} / \mathrm{mL}$.

The above results were surprising considering previous in vitro studies showed an active and quick uptake of doxHCl molecules by the PEGlated nanoparticles that was limited only by the maximal drug loading potential. However, these differences could be explained by the presence of dissolved plasma proteins, which were not present during the in vitro studies. These proteins have a tendency to reversibly adsorb to hydrophobic nanoparticle surface despite the presence of hydrophilic PEG polymers on the nanoparticles, which was only around $2.5 \%$ by total nanoparticle weight and was not effective for shielding the entire nanoparticle surface from the plasma proteins. Thus, any drug molecules that came into contact with nanoparticles was likely to first encounter an outer shell of plasma proteins and was preferentially bound by these proteins until gradually, available protein binding sites were saturated and excess drug could access and bind to nanoparticle itself.

Both percent plasma drug concentration reduction and drug loading results also suggested the PEGylated nanoparticles were not effective around the normal therapeutic concentration of $50 \mu \mathrm{g} / \mathrm{mL}$, which was actually desirable considering a minimum therapeutic concentration was needed for treatment. At $4 \mathrm{X}$ the normal therapeutic concentration and above, the PEGylated nanoparticle could be very effective for treating potential drug overdose considering the high drug loading and quick uptake (30minute incubation time.) Although not tested here, drug loading results indicated that more drug uptake into the nanoparticles were possible considering these particles could reach up to $6 \%$ drug loading. Although only doxHCl was tested in this study, these nanoparticles could be effective for the broad category of small molecule drugs, including vinblastine sulfate, to reduce potential toxicity.

\section{Conclusions}

Anionic borates demonstrated great potential as model lipophilic anions with successful extraction of a model hydrophilic drug, doxorubicin hydrochloride $(\mathrm{doxHCl})$, from aqueous to the organic phase via ion pairing. Of the borates studied. KTpCIPB was most suitable for nanoparticle incorporation and was incorporated into regular or PEGylated nanoparticles at high concentrations to form spherical nanoparticles with PEG localized on the outer shell according to XPS.

In vitro incubation drug loading procedure, tested with these specially formulated nanoparticles, successfully extracted two model drugs, doxorubicin hydrochloride and vinblastine sulfate, into the particles at high concentrations. This drug loading process was optimized using polymers with high acid number (more negative charges for ion complexing), maximizing the KTpCIPB loading into nanopartilces, increasing 
incubation temperature, maximizing drug:nanoparticle (wt/wt) ratio, and using PEGylated nanoparticles. These improvements enhanced loading by potentially incrasing the density of available negative charges in nanoparticles for ion pairing, introducing potential hydrophobic interaction between drug and KTpCIPB molecules to stabilize and enhance loading, and preventing or reducing potential particle aggregation through PEGylation.

Plasma doxHCl uptake, using optimized PEGylated nanoparticle, was negligible when the normal therapeutic dose of doxHCl was administered, which was desirable. However, such particles effectively reduced both plasma drug concentration and achieved high drug loading when $4 \mathrm{X}$ the normal therapeutic concentration was administered. This promising result suggested potential application of these nanoparticles as treatment for drug overdose of small molecule drugs with similar properties to dox $\mathrm{HCl}$.

Overall, the combination of KTpCIPB-loaded PEGylated nanoparticles and an optimized absorption/extraction loading process could significantly enhance active drug loading and provide a viable alternative to entrapment and conjugation-based drug loading methods.

Table 3. 1. Properties of the borate compounds studied

\begin{tabular}{|l|c|c|c|}
\hline \multicolumn{1}{|c|}{ Compounds } & Molecular formula & MW (g/mol) & LogP \\
\hline Potassium tetrakis(2-thienyl)borate (KTTB) & C16H12BS4K & 382.44 & 6.152 \\
\hline Sodium tetraphenyl borate (NaTPB) & C24H20BNa & 342.23 & 7.568 \\
\hline $\begin{array}{l}\text { Potassium tetrakis[3,5-bis- } \\
\text { chlorophenyl)borate (KTpClPB) }\end{array}$ & C24H16BC14K & 496.12 & 10.42 \\
\hline $\begin{array}{l}\text { (trifluoromethyl)phenyl]borate } \\
\text { (KTBTFMPB) }\end{array}$ & C32H12BF24K & 902.32 & 14.632 \\
\hline
\end{tabular}

\#LogP values calculated by ChemDraw (octanol/water partition coefficient)

Table 3. 2. KTpCIPB extraction from O/W nanoparticles with $40 \mathrm{mg}$ total polymer/batch

\begin{tabular}{|c|c|c|c|c|}
\hline Nanoparticle & $\begin{array}{c}\text { Theoretical KTpCIPB } \\
(\%)\end{array}$ & Incubation & Actual loading (\%) & $\begin{array}{l}\text { Encapsulation } \\
\text { Efficiency (\%) }\end{array}$ \\
\hline 7:1-502H/PEG-PLA & None & None & 0 & 0 \\
\hline 7:1-502H/PEG-PLA & 10.7 & None 4. & $19 \pm 0.04$ & 39.12 \\
\hline $7: 1-502 \mathrm{H} / \mathrm{PEG}-\mathrm{PLA}$ & $10.7 \mathrm{~b}$ & 1 hour in DIW & $4.02 \pm 0.1$ & 37.54 \\
\hline 7:1-502H/PEG-PLA & 10.7 & $30 \mathrm{~min}$ in & $4.43 \pm 0.10$ & 41.36 \\
\hline
\end{tabular}

Table 3. 3. Properties of different PLGA tested

\begin{tabular}{|c|c|c|c|}
\hline PLGA polymer & $\begin{array}{c}\text { Molecular Weight } \\
\text { (g/mol) }\end{array}$ & Viscosity & Acid number \\
\hline Resomer® RG 502H & $\sim 15,000$ & $0.2 \mathrm{dl} / \mathrm{g}$ & $\mathrm{min} .6 \mathrm{mg} \mathrm{KOH} / \mathrm{g}$ \\
\hline Resomer® RG 503H & $\sim 38,000$ & $0.34 \mathrm{dl} / \mathrm{g}$ & $\mathrm{min} .3 \mathrm{mg} \mathrm{KOH} / \mathrm{g}$ \\
\hline Resomer® RG 503 & $\sim 45,000$ & $0.42 \mathrm{dl} / \mathrm{g}$ & $\begin{array}{c}\text { End-capped }(\mathrm{max} .1 \mathrm{mg} \\
\mathrm{KOH} / \mathrm{g}\end{array}$ \\
\hline
\end{tabular}

Table 3. 4. Atomic \% composition of C1s, O1s, and N1s; of XPS C1s core-level spectra of various nanoparticle samples

\begin{tabular}{|l|c|c|c|}
\hline \multirow{2}{*}{ Nanoparticle samples } & \multicolumn{3}{|c|}{ Atomic composition (\%) } \\
\cline { 2 - 4 } & C1s & O1s & N1s \\
\hline PLGA & 43.6 & 56.4 & 0.0 \\
\hline PEG-PLA & 48.6 & 51.4 & 0.0 \\
\hline PLGA:PEG-PLA & 45.8 & 54.2 & 0.0 \\
\hline PLGA + KTpClPB & 45.4 & 54.6 & 0.1 \\
\hline PLGA:PEG-PLA +KTpClPB & 51.2 & 48.8 & 0.0 \\
\hline PLGA:PEG-PLA + KTpClPB + doxorubicin & 52.1 & 47.9 & 0.0 \\
\hline
\end{tabular}

Table 3.5 Decomposition of $\mathrm{C}_{18}$ peak (\% envelope ratio) of nanoparticle samples

\begin{tabular}{|c|c|c|c|c|}
\hline \multirow[b]{2}{*}{$\begin{array}{l}\text { Nanoparticle } \\
\text { Samples }\end{array}$} & \multicolumn{4}{|c|}{ Carbon peak components } \\
\hline & $\begin{array}{c}\text { C-C, C-H } \\
\text { (hydrocarbon, } \\
285 \mathrm{eV}) \\
\end{array}$ & $\begin{array}{c}\text { C-O } \\
\text { (PEG, } \\
\text { 286. eV) }\end{array}$ & $\begin{array}{c}\text { C-O } \\
\text { (polyester, } \\
287.2 \mathrm{eV} \text { ) }\end{array}$ & $\begin{array}{c}\mathrm{O}=\mathrm{C}-\mathrm{O}-\mathrm{C} \\
(\text { carboxyl, } 289.2 \mathrm{eV})\end{array}$ \\
\hline PLGA & 26.91 & 10.35 & 28.69 & 33.04 \\
\hline PEG-PLA & 30.62 & 18.04 & 25.63 & 25.70 \\
\hline PLGA:PEG-PLA & 27.79 & 11.15 & 29.58 & 31.49 \\
\hline PLGA + KTpClPB & 29.50 & 11.02 & 27.38 & 32.09 \\
\hline $\begin{array}{l}\text { PLGA:PEG-PLA + } \\
\text { KTpClPB }\end{array}$ & 40.47 & 32.79 & 11.70 & 15.04 \\
\hline $\begin{array}{l}\text { PLGA:PEG-PLA + } \\
\text { KTpCIPB +doxorubicin }\end{array}$ & 42.99 & 34.20 & 10.49 & 12.31 \\
\hline
\end{tabular}


Table 3. 6. Drug loading and encapsulation efficiency for vinblastine sulfate

\begin{tabular}{|c|c|c|}
\hline Nanoparticle formulation & Drug loading (\%) & E.E. (\%)* \\
\hline 502H:PEG(7:1)-room temp & $3.77 \pm 0.54$ & 22.6 \\
\hline 502H:PEG(7:1)-37oC & $5.98 \pm 0.90$ & 35.9 \\
\hline 502H:PEG(7:1)-KTpClPB & $8.98 \pm 0.76$ & 53.9 \\
\hline
\end{tabular}

*E.E.: encapsulation efficiency<smiles>[2H]C1c2cccc(OC)c2C(O)C2C=C([C@@H](C)C[C@@H](C[C@@H](N)CO)OCC)C(CC(O)C(=O)CO)=CC12</smiles>

Figure 3. 1. Structure of doxorubicin hydrochloride (doxHCl).
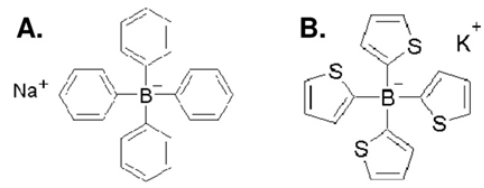

C.

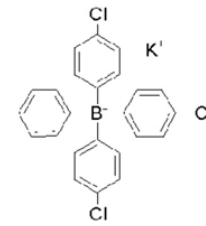

D.

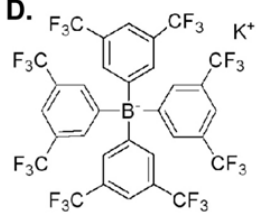

Figure 3. 2. Structures of the borate compounds studied, (A) sodium tetraphenylborate (NaTPB), (B) potassium tetrakis(2-thienyl)borate (KTTB), (C) potassium tetrakis(4chlorophenyl)borate (KTpClPB), and (D) potassium tetrakis[3,5-

bis(trifluoromethyl)phenyl]borate (KTBTFMPB).

\section{Solubility of borate compound in organic solvents}

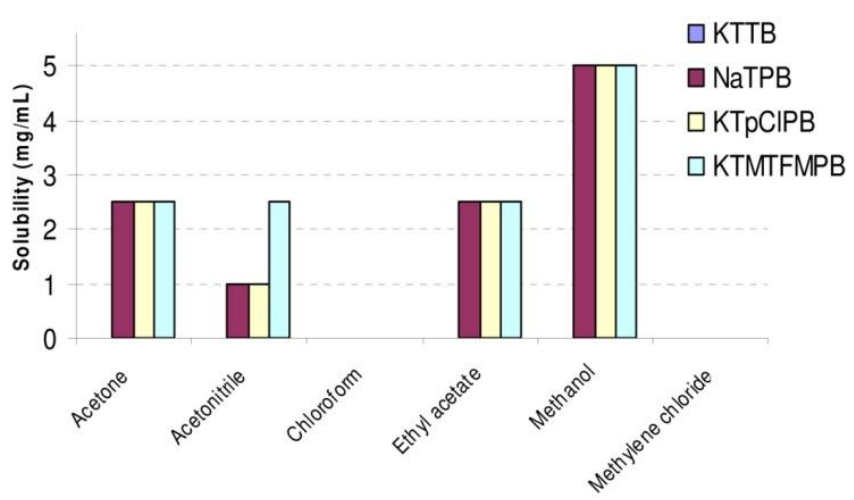

Figure 3. 3. Borate solubilities in various organic solvents. 

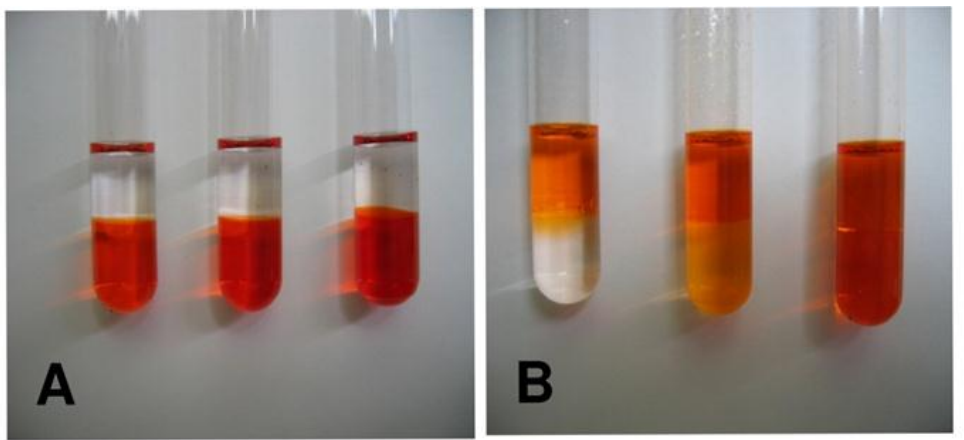

Figure 3. 4. Doxorubicin hydrochloride extraction into blank ethyl acetate at differe aqueous dox $\mathrm{HCl}$ concentrations (A) or at 1:0.5, 1:1, and 1:1.5 molar ratios (left to ri photo B) of representative borate:doxHCl.

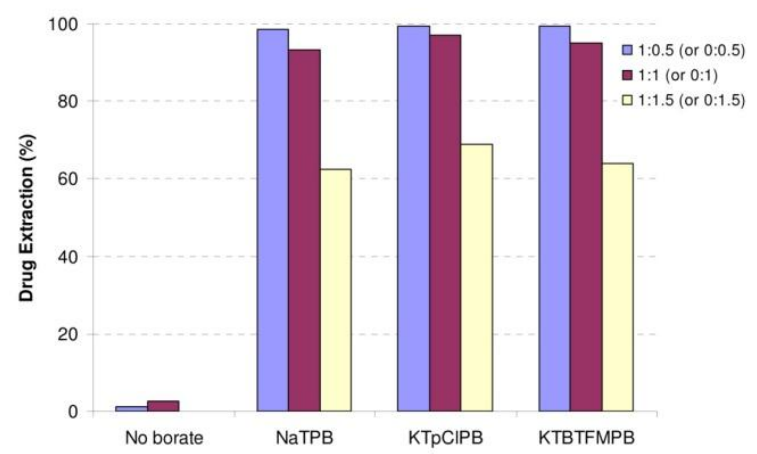

Figure 3. 5. Percent dox $\mathrm{HCl}$ extraction into ethyl acetate phase with and without dissolved borates. Three different molar ratios $(1: 0.5,1: 1$, and 1:1.5) of borate:doxHCl were studied.

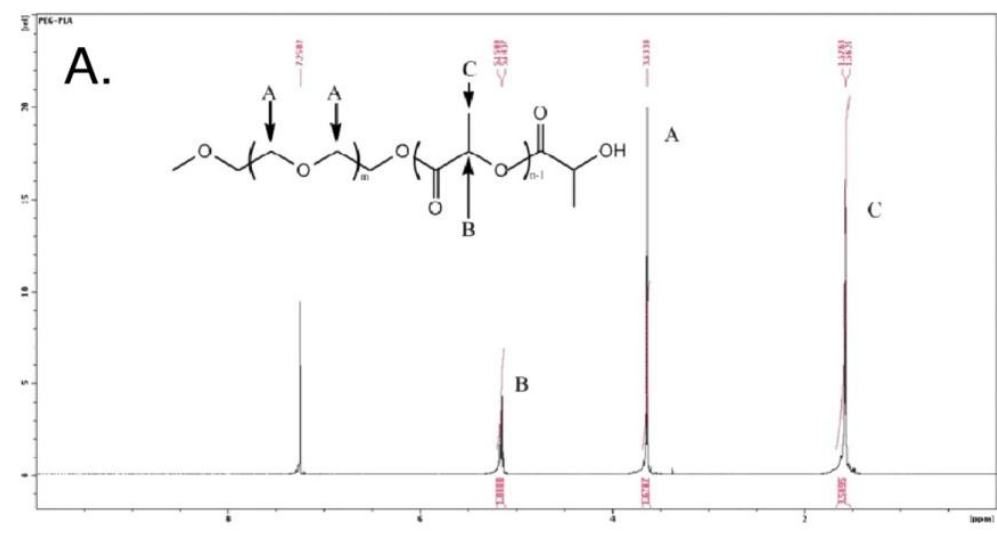

B.

\begin{tabular}{|c|c|c|c|}
\hline $\begin{array}{l}\text { PEG MW } \\
(\mathrm{g} / \mathrm{mol})\end{array}$ & $\begin{array}{c}\text { PEG-PLA theoretical } \\
\text { MW }^{1}(\mathrm{~g} / \mathrm{mol})\end{array}$ & $\begin{array}{c}\text { PEG-PLA calculated } \\
\mathrm{MW}^{2}(\mathrm{~g} / \mathrm{mol})\end{array}$ & $\begin{array}{c}\text { PEG-PLA calculated } \\
\mathrm{MW}^{3}(\mathrm{~g} / \mathrm{mol})\end{array}$ \\
\hline 5,000 & 25,000 & $19,408.54$ & $22,689.8$ \\
\hline
\end{tabular}

Figure 3. 6. (A) ${ }^{1} \mathrm{H}-\mathrm{NMR}$ spectrum of methoxyPEG-PLA copolymer in deuterated chloroform and (B) associated table with theoretical and calculated molecular weights of the copolymer based on peak area ratios. 


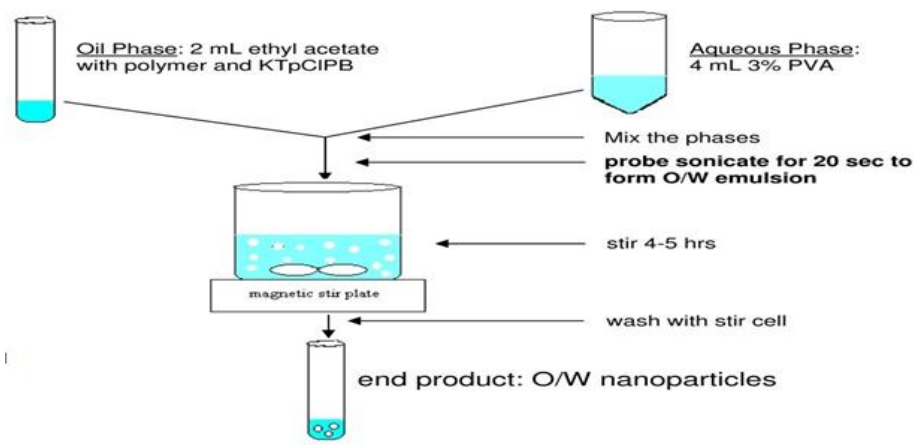

Figure 3. 7. General oil-in-water $(\mathrm{O} / \mathrm{W})$ blank nanoparticle formulation procedure.

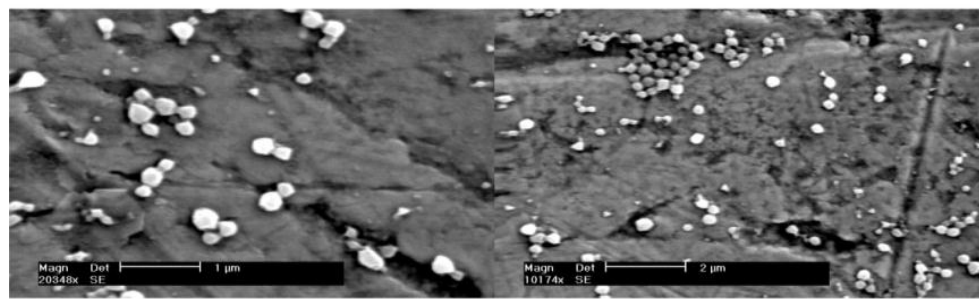

Figure 3. 8. SEM images of KTpCIPB-loaded $\mathrm{O} / \mathrm{W}$ nanoparticles at different magnifications (Philips XL30 FEG SEM).

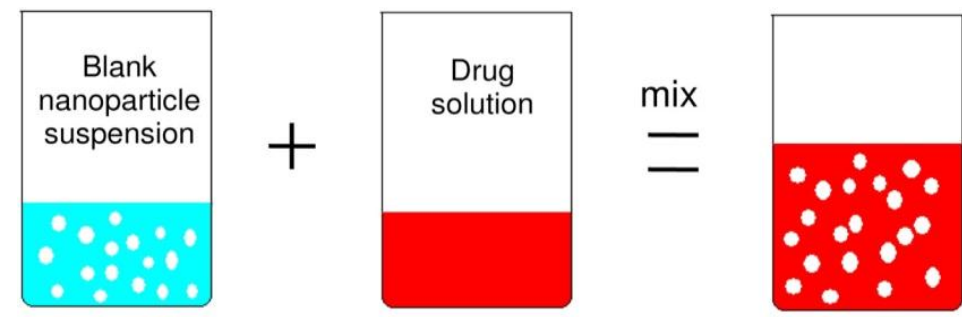

Figure 3. 9. General drug extraction/absorption loading into nanoparticle process.

A.

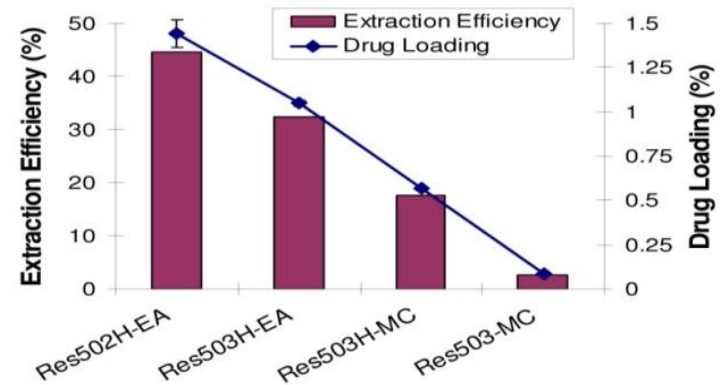

B.

\begin{tabular}{ccccc}
\hline Sample & $\begin{array}{c}\text { Zeta potential-before } \\
\text { extraction }(\mathbf{m V})\end{array}$ & $\begin{array}{c}\text { Zeta potential-after } \\
\text { extraction }(\mathbf{m V})\end{array}$ & $\begin{array}{c}\text { Particle } \\
\text { size }(\mathbf{n m})\end{array}$ & Polydispersity \\
\hline Res502H-EA & -30.3 & -14.7 & 179.5 & 0.155 \\
Res503H-EA & -25.5 & -14.5 & 189.8 & 0.133 \\
Res503H-MC & -33.7 & -23.1 & 229.4 & 0.108 \\
Res503-MC & -36.2 & -18.9 & 240.2 & 0.159 \\
\hline
\end{tabular}

Figure 3. 10. Dox $\mathrm{HCl}$ extraction/absorption into nanoparticles made with different polymers with extraction efficiency and drug loading (A) and associated table of zeta potential and size of drug-loaded nanoparticles (B). 


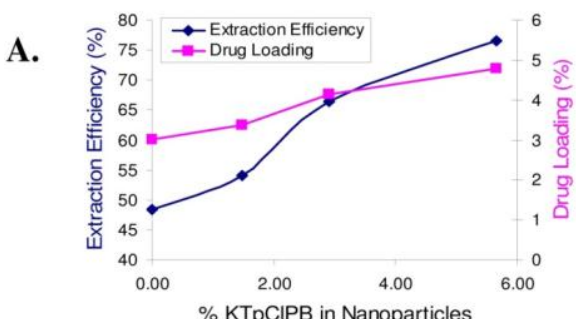

B.

\begin{tabular}{ccccc}
\hline $\begin{array}{c}\text { Theoretical \% } \\
\text { KTpCIPB } \\
\text { present }\end{array}$ & $\begin{array}{c}\text { Theoretical amt } \\
\text { KTpCIPB/batch } \\
\text { NP }(\mathbf{m g})\end{array}$ & $\begin{array}{c}\text { Zeta potential- } \\
\text { before extraction } \\
(\mathbf{m V})\end{array}$ & $\begin{array}{c}\text { Zeta potential- } \\
\text { after extraction } \\
(\mathbf{m V})\end{array}$ & $\begin{array}{c}\text { Particle } \\
\text { size }(\mathbf{n m})\end{array}$ \\
\hline 0.00 & 0 & -30.4 & -21.3 & 182.5 \\
1.48 & 1.2 & -33.8 & -18 & 161.7 \\
2.91 & 2.4 & -37.2 & -15.7 & 142.6 \\
5.66 & 4.8 & -35.4 & -17.3 & 117.1 \\
\hline
\end{tabular}

Figure 3. 11. DoxHCl extraction/absorption into nanoparticles made with different polymers with extraction efficiency and drug loading (A) and associated table of zeta potential and size of drug-loaded nanoparticles (B).

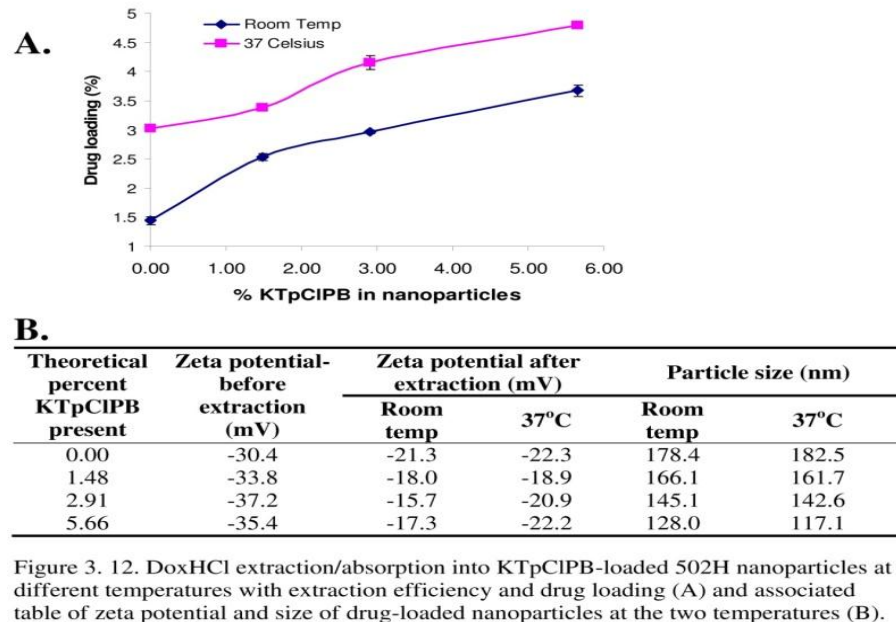

A.

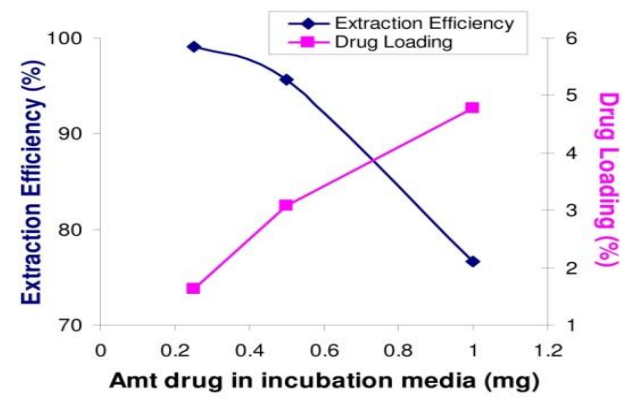

B.

\begin{tabular}{cccc}
\hline $\begin{array}{c}\text { DoxHCl in } \\
\text { incubation } \\
\text { media (mg) }\end{array}$ & $\begin{array}{c}\text { Zeta potential- } \\
\text { before } \\
\text { extraction }(\mathbf{m V})\end{array}$ & $\begin{array}{c}\text { Zeta potential- } \\
\text { after extraction } \\
(\mathbf{m V})\end{array}$ & $\begin{array}{c}\text { Particle } \\
\text { size } \\
(\mathbf{n m})\end{array}$ \\
\hline 0.25 & -35.4 & -24.5 & 120.2 \\
0.5 & -35.4 & -26.6 & 123.0 \\
1 & -35.4 & -22.1 & 117.1 \\
\hline
\end{tabular}

Figure 3. 13. DoxHCl extraction/absorption into KTpClPB-loaded 502 $\mathrm{H}$ nanoparticles at different amounts of dox $\mathrm{HCl}$ to $15 \mathrm{mg}$ nanoparticles with extraction efficiency and drug loading (A) and associated table of zeta potential and size of drug-loaded nanoparticles at the two temperatures (B) 
A.

B.

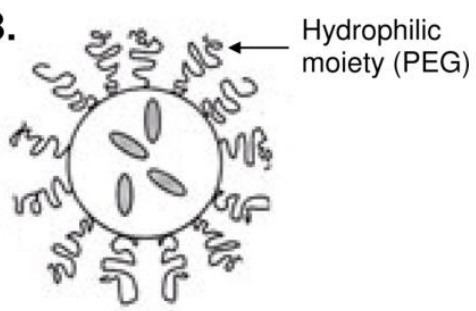

Figure 3. 14. Schematic of (A) regular vs. (B) PEGylated nanoparticles.

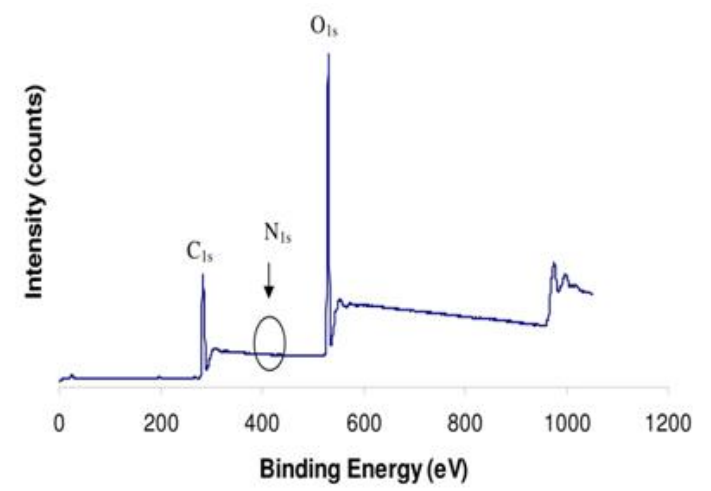

Figure 3. 15. General XPS survey scans of PEGylated nanoparticles with characteristic peaks for carbon and oxygen atoms and nitrogen at $400 \mathrm{eV}$.
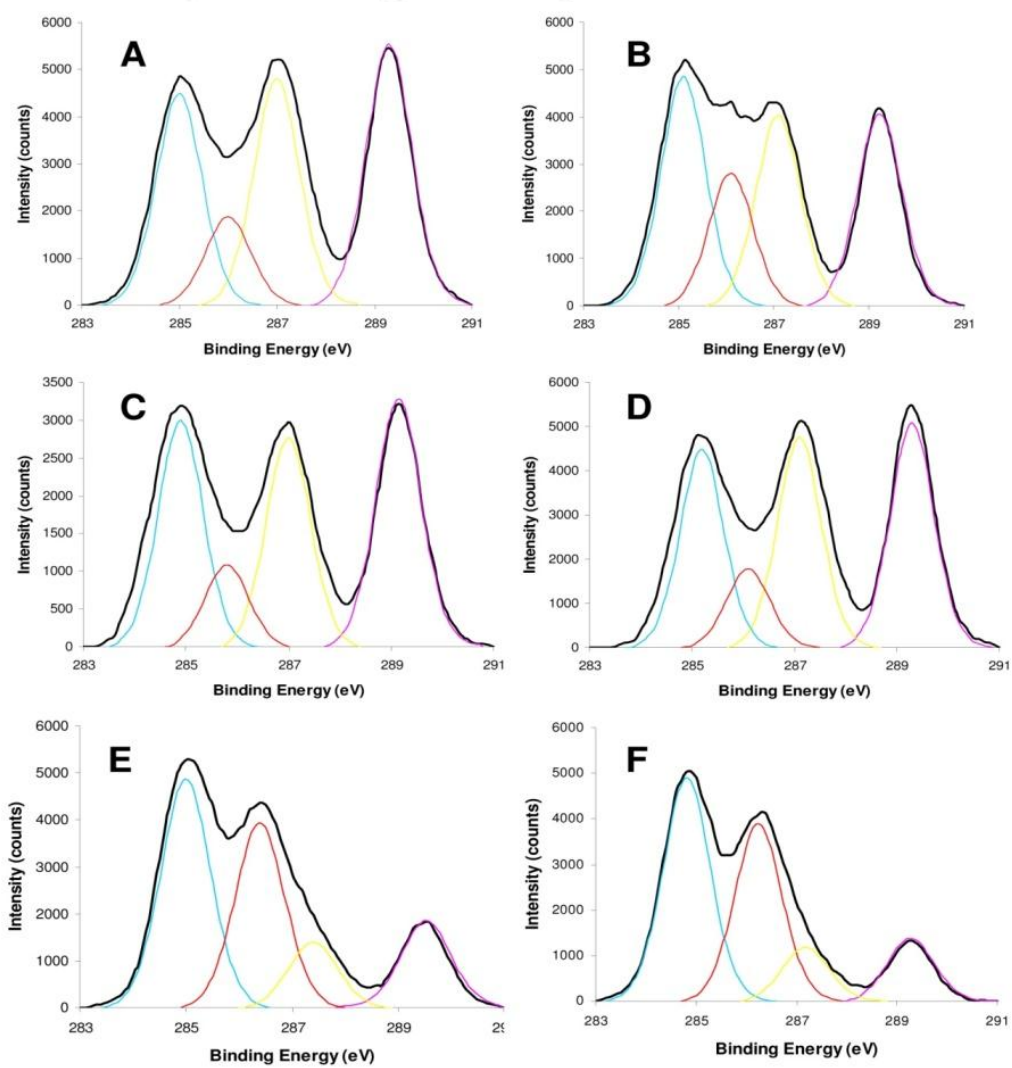

Figure 3. 16. Synthetic XPS peak fits for $\mathrm{C}_{1 \mathrm{~s}}$ envelopes of PLGA (A), PEG-PLA (B), PLGA:PEG-PLA ( 7:1, wt/wt) (C), PLGA + KTpCIPB (D), PLGA:PEG-PLA + KTpCIPB (E), and PLGA:PEG-PLA + KTpCIPB+doxorubicin (F) nanoparticles. 

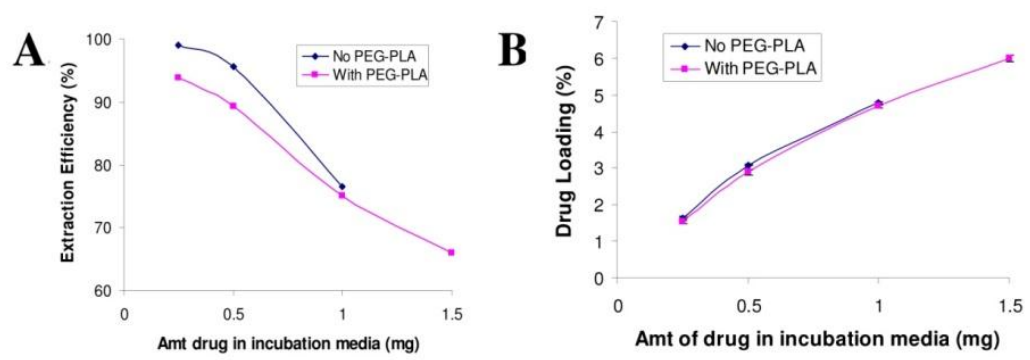

C.

\begin{tabular}{|c|c|c|c|c|}
\hline \multirow{2}{*}{$\begin{array}{l}\text { DoxHCl in } \\
\text { incubation } \\
\text { media (mg) }\end{array}$} & \multicolumn{2}{|c|}{ Zeta potential $(\mathrm{mV})$} & \multicolumn{2}{|c|}{ Particle size (nm) } \\
\hline & $\begin{array}{c}\text { Non- } \\
\text { PEGylated }\end{array}$ & PEGylated & $\begin{array}{c}\text { Non- } \\
\text { PEGylated }\end{array}$ & PEGylated \\
\hline 0 (blank) & -35.4 & -35.7 & 132.0 & 128.8 \\
\hline 0.25 & -24.5 & -38.7 & 120.2 & 115.0 \\
\hline 0.5 & -26.6 & -33.5 & 123.0 & 112.8 \\
\hline 1 & -22.1 & -24.0 & 117.1 & 230.1 \\
\hline 1.5 & NA* & -26.4 & NA* & 322.1 \\
\hline
\end{tabular}

Aggregation of non-PEGylated nanoparticles

Figure 3. 17. DoxHCl extraction/absorption into regular or PEGylated nanoparticles at different ratios of doxHCl to $15 \mathrm{mg}$ nanoparticles with extraction efficiency (A) and drug loading (B) and associated table of zeta potential and size of drug-loaded regular or PEGylated nanoparticles (C).
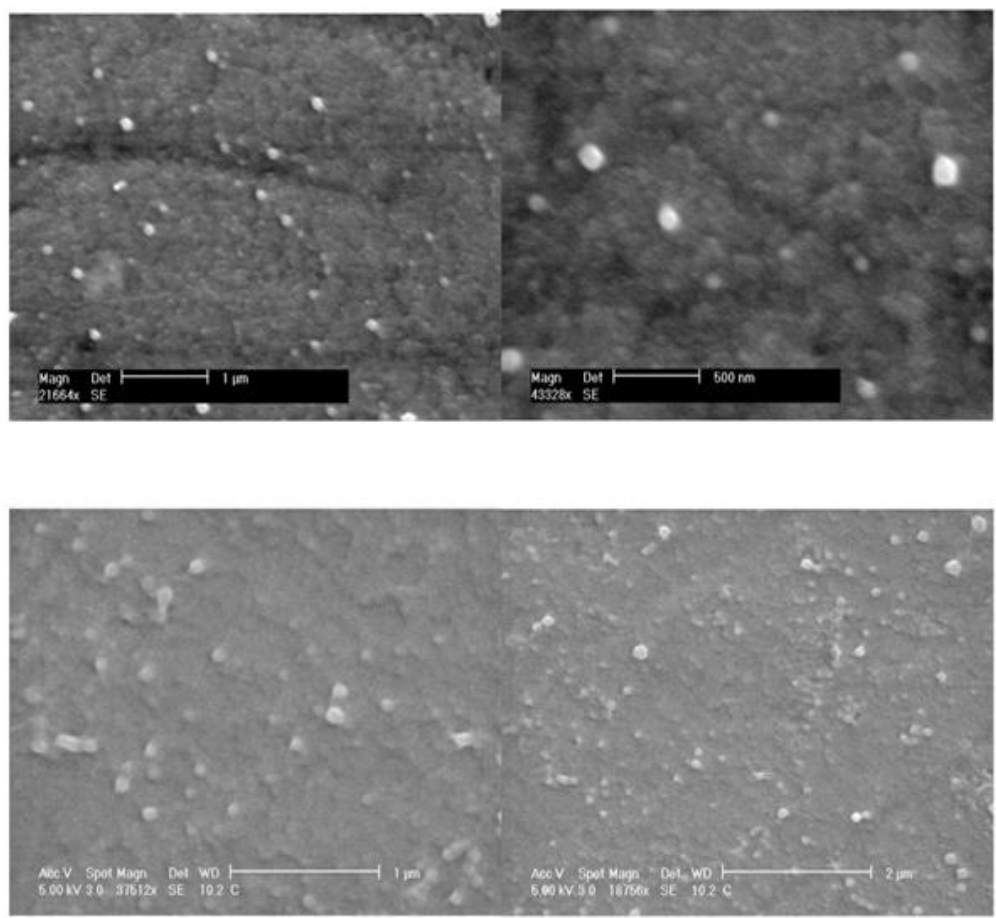

Figure 3. 18. SEM of non-PEGylated (top row) and PEGylated (bottom row) nanoparticles with $\mathrm{KTpClPB}$ and doxHCl loaded. 


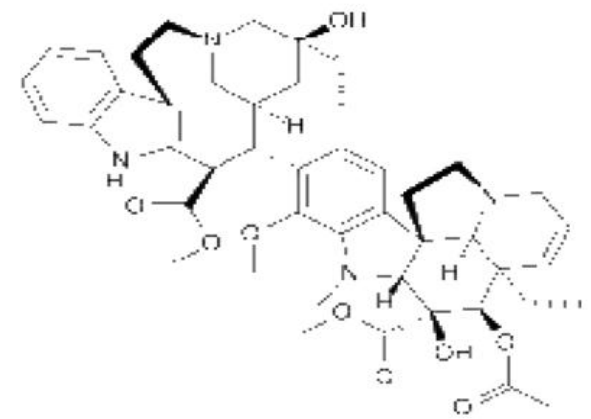

Figure 3. 19. Structure of vinblastine sulfate.

\section{References:}

[1] Arias, J.L.,F.Linares-Molinero, et al. (2008). "Study of carbonyl iron/poly (butylcyanoacrylate) (core/shell) particles as anticancer drug delivery systems - Loading and release properties. "European Journal of Pharmaceutical Sciences 33(3): 252-261.

[2] Arias, J.L.,M.A.Ruiz, et al. (2008). "Poly(alkylcyanoacrylate) colloidal particles as vehicles for antitumour drug delivery: A comparative study. "Colloids and Surfaces B-Biointerfaces 62(1): 64-70

[3] Asuri, P.,S.S.Karajanagi, et al. (2007). "Enhanced stability of enzymes adsorbed onto naoparticles."journal of Nanoscience of Nanotechnology 7(4-5):1675-1678

[4] Blasi, P., S.S.D'Souza, et al,(2005). "Plasticizing effect of water on poly(lactide-co-glydolide).”Journal of Controlled Release 108 (1):1-9.

[5] Cai, C., U.Bakowsky, et al. (2008). "Charged nanoparticles as protein delivery systems: A feasibility study using lysozyme as model protein.”Eur J Pharm Biopharm 69(1):31-42.

[6] Dong, Y.C.and S.S.Feng(2004), "Methoxy poly(ethylene glycol)-poly(lactide)(MPEG-PLA) nanoparticles for controlled delivery of anticancer drugs. "Biomaterials 25(14):2843-2849.

[7] Gaertner, L.S.C.L. Murray, et al (1998). "Transepithelial transport of nicotine and vinblastine in isolated malpighian tubules of the tobacco hornworm (Manduca sexta)suggests a P-glycoprotein-like mechanism. "Journal of Experimental Biology 210(18):26372649.

[8] Galindo-Rodriguez, S.,E.Allemann, et al.(2004). "Physicochemical parameters associated with nanoparticle formation in the salting -out, emulsification-diffusion, and nanoprecipitation methods."Pharmaceutical Research 21(8):1428-1439.

[9] Ganjali, M.R.,P.Norouzi, et al, (2006). "Application of 8-amio-N-(2-hydroxybenzylidene) naphthyl amine as a neutral ionophore in the construction of a lanthanum ion-selective sensor."Analytica Chimica Acta 576(2):275-282.

[10] Gou, M.L.,M.J.Huang, et al.(2007). "Preparation of anionic poly(epsilon-caprolactone)-poly(ethylene glycol)poly (epsiloncaprolactone) copolymedric nanoparticles as basic protein antigen carrier."Growth Factor 25:202-208.

[11] Gupta, V.K., A.K.Singh, et al. (2008), "Electroanalytical performance of a terbium(III)-selective sensor based on a neutral ionophore in environmental and medicinal samples." Analytical and Bioanalytical Chemistry 390(8):2171-2181.

[12] Heald, C.R.S.Stolnik, et al (2002). "Poly(lactic acid)-poly(ethylene oxide)(PLA-PEG) nanoparticles: NMR studies of the central solidlike PLA core and the liquid PEG corona. "Langmuir 18(9):3669-3675.

[13] Jung.T.A.Breitenbach, et al(2000). "Sulfobutylated poly(vinyl alcohol)-graft-poly(lactide-co-glycolide)s facilitiate the preparation of small negative charged biodegradable nanospheres.”Journal of Controlled Release 67(2-3):157-169.

[14] Kang, J.C.and S.P.Schwendeman (2003). "Determination of diffusion coefficient of a small hydrophobic probe in poly(lactide-coglycolide)microparticles by laser scanning confocal microscopy.”Macromolecules 36(4):1324-1330.

[15] Krauel. K., N.M.Davies, et al.(2005)."Using different structure types of microemulsions for the preparation of poly(alkylcyanoacrylate)nanoparticles by interfacial polymerization.’Journal of Controlled Release 106(1-2):76-87.

[16] Missirlis, D.,R.Kawamura, et al.(2006). "Doxorubicin encapsulation and diffusional release from stable, polymeric, hydrogel nanoparticles, "European Journal of Pharmaceutical Sciences 29(2):120-129.

[17] Olivier, J.C.R.Huertas,et.al.(2002).”Synthesis of pegylated immunonanoparticles. "Pharmaceutical Research 19(8)1137-1143

[18] Park,K.(2007)."Nanotechnology:What it can do for drug delivery.”Journal of Controlled Release 120(1-2):1-3

[19] Pourcelle,V.,S.Devouge,et al (2007).’PCL-PEG-Based nanoparticles grafted with GRGDS peptide: Preparation and surface analysis by XPS."Biomacromolecules 8:3977-3983.

[20] Scholes, P.D.,A.G.A.Coombes, et al.(1999)."Detection and determination of surface levels of poloxamer and PVA surfactant on biodegradable nanospheres using SSIMS and XPS.”Journal of Controlled Release 59(3):261-278.

[21] Shakesheff, K.M.,C.Evora, et al(1997). "The adsorption of poly(vinyl alcohol)to biodegradable microparticles studied by x-ray photoelectron spectroscopy (XPS).” Journal of Colloid and Interface Science 185(2):538-547.

[22] Soma, C.E.,C.Dubernet, et al.(2000). "Investigation of the role of macrophages on the cytotoxicity of doxorubicin and doxorubicinloaded nanoparticles on M5076 cells in vitro.”Journal of Controlled Release 68(2):283-289.

[23] Soppimath, K.S., T.M.Aminabhavi, et al.(2001). "Biodegradable polymeric nanoparticles as drug delivery devices."Journal of Controlled Release 70(1-2):1-20.

[24] Wong, H.L., R.Bendayan, et al. (2004). "Development of solid lipid nanoparticles containing lonically complexed chemotherapeutive drugs and chemosensitizers."Journal of Pharmaceutical Sciences 93(8).1993-2008.

[25] Yang,Y.Y., Y.Wang, et.al(2006)"Polymeric core-shell nanoparticles for therapeutics, "Clinical and Experimental Pharmacology and Physiology 33(5-6):557-562.

[26] Yoo,H.S., J.E.Oh,et al. (1999). "Biodegradable nanoparticles containing doxorubicin-PLGA conjugate for sustained release. "Pharmaceutical Research 16(7):1114-1118.

[27] Zobel, H.P., A.Zimmer, et al.(1999). "Evaluation of aminoalkylmethacrylate nanoparticles as colloidal drug carrier systems. Part I:synthesis of monomers, dependence of the physical properties on the polymerization methods. "European Journal of Pharmaceutics and Biopharmaceutics 47(3):203-213. 